\title{
DO PSYCHOLOGICAL BARRIERS EXIST IN LATIN AMERICAN STOCK MARKETS?
}

\section{¿EXISTEN BARRERAS PSICOLÓGICAS EN LOS MERCADOS BURSÁTILES LATINOAMERICANOS?}

\section{JÚLIO LOBÃO*}

Universidade do Porto - Faculdade de Economia do Porto

\author{
NATÉRCIA FORTUNA* \\ Universidade do Porto - Faculdade de Economia do Porto
}

\section{FRANKLIN SILVA*}

Universidade do Porto - Faculdade de Economia do Porto

\begin{abstract}
We examine for the first time the major stock markets of eight Latin American countries for indication of psychological barriers at round numbers. We test for uniformity in the trailing digits of the indices and use regression and GARCH analysis to assess the differential impact of being above or below a possible barrier. The Chilean stock market seems to be significantly different from its counterparts as it is the only one that showed virtually no signs of psychological barriers. There is mild to strong evidence of barriers in the remaining markets. These findings challenge the notion that most Latin American markets are unpredictable and lend credit to the claim that technical analysis strategies can be useful in some of these markets.
\end{abstract}

Key words: Latin American markets, psychological barriers, stock market indices, market psychology, round numbers.

JEL-Codes: G11, G12, G14, G15.

* Universidade do Porto - Faculdade de Economia do Porto (University of Porto - School of Economics and Management). Rua Dr. Roberto Frias, 4200-464. Porto - Portugal. T: +351 22225571100; F: +351225505050. E-mails: jlobao@fep.up.pt, nfortuna@fep.up.pt, 110401171@fep.up.pt 


\section{Resumen}

Examinamos por primera vez los principales mercados bursátiles de ocho países latinoamericanos para indicación de barreras psicológicas en los números redondos. Probamos la uniformidad en los dígitos finales de los índices y usamos regresións y análisis GARCH para evaluar el impacto diferencial de estar por encima o debajo de una posible barrera. El mercado de valores chileno parece ser significativamente diferente de sus contrapartes, ya que es el único que prácticamente no mostró señales de barreras psicológicas. Hay evidencia leve a fuerte de barreras en los restantes mercados. Estos hallazgos desafían la noción de que la mayoría Los mercados latinoamericanos son impredecibles y otorgan crédito al reclamo que las estrategias de análisis técnico pueden ser útiles en algunos de estos mercados.

Palabras clave: Mercados latinoamericanos, barreras psicológicas, indices bursátiles, psicología de mercado, números redondos.

Clasificación JEL: G11, G12, G14, G15.

\section{INTRODUCTION}

Market practitioners and journalists often refer to the existence of psychological barriers in stock markets. Many investors believe that round numbers serve as barriers, and that prices may resist crossing these barriers. Moreover, the use of technical analysis is based on the assertion that traders will "jump on the bandwagon" of buying (selling) once the price breaks up (down) through a "psychologically important level" thus suggesting that the crossing of one of these barriers may push the prices up (down) more than otherwise warranted. Frequently used phrases by the business press such as "support levels" and "resistance levels" imply that, until such time as an important barrier is breached, increases and decreases in the prices may be restrained.

The impact of such kind of psychological barriers in investors' decisions has been studied since the 1990's for a variety of asset classes, from exchange rates with De Grauwe and Decupere (1992) to stock options with Jang et al. (2015). The evidence of psychological barriers on stock market indices suggests some significant impacts of this phenomenon in the returns and variances in different geographies and periods (e.g., Koedijk and Stork, 1994; Cyree et al., 1999; Bahng, 2003; Woodhouse et al., 2016).

This article examines the existence of psychological barriers at round numbers in the major stock market indices of eight Latin American countries: Argentina (MERVAL), Brazil (BOVESPA), Chile (IPSA), Colombia (COLCAP), Mexico (IPC), Panama (BVPSI), Peru (IGBVL), and Venezuela (IBVC). To the best of our knowledge, none of these markets has ever been analysed with this purpose. 
The anchoring effect, a well-known behavioral bias firstly identified by Tversky and Kahneman (1974), is the main explanation for the existence of psychological barriers in financial markets. Individuals, when performing an estimation in an ambiguous situation, tend to fixate ('to anchor') on a salient number even if that number is irrelevant for the estimation. The anchoring on round numbers is important for its great explanatory power of some of the features commonly associated to financial markets. It may help to understand, for example, the excessive price volatility (Westerhoff, 2003), the momentum effect (George and Hwang, 2004), or even the emergence of speculative bubbles (Shiller, 2015).

Of course, behavioral biases are not the only reason why barriers could exist. For example, the fact that option exercise prices also are usually round numbers may be an additional explanation for the phenomenon.

The existence of psychological barriers points to some level of predictability in stock markets and thus may potentially lead to abnormal risk-adjusted returns. Hence empirical evidence for the existence of psychological barriers is not only of interest to practitioners who are looking for profitable strategies but it also represents a contribution to the literature on market anomalies.

To investigate the existence of psychological barriers, we conduct tests for positional and transgressional effects. We test for uniformity in the trailing digits of the stock indices and use regression and GARCH analysis to assess the differential impact of being above or below a possible barrier. The results obtained reveal substantial differences in the incidence of psychological barriers on the markets of the sample. The Chilean stock market is the only one that shows virtually no signs of psychological barriers. There is mild evidence of barriers in the stock markets of Brazil, Colombia and Venezuela and strong indication of psychological barriers in the stock markets of Argentina, Mexico, Panama and Peru. These results are difficult to reconcile with the notion that capital markets are unpredictable and lend support to the claim that trading strategies based on price support and resistance levels can be useful at least in some Latin American stock markets.

This article is organized in as follows. Section 2 reviews the empirical evidence regarding psychological barriers. Section 3 presents the data and methodologies used in this paper. Section 4 presents the empirical results. Section 5 offers conclusions.

\section{PREVIOUS FINDINGS}

Donaldson (1990a, 1990b) and De Grauwe and Decupere (1992) were the first to study the phenomenon of psychological barriers and showed that round numbers are indeed of special importance for investors in the stock market and in the foreign exchange markets, respectively. From then on, several other studies followed, focusing not only on different geographies and periods, but also on different asset classes, such as bonds, commodities and derivatives. 
However, to date, little research has been conducted on the existence of psychological barriers in emerging markets. Bahng (2003) constitutes a noteworthy exception. The author considered seven major Asian stock market indices to show that the Taiwanese index did possess significant price barrier effects and that the price level distributions of the Taiwanese, Indonesian, and Hong Kong indices were not uniform.

Cai, Cai and Keasey (2007) studied the price behavior of 1,050 Chinese stocks and conclude that the digits 0 and 5 constituted significant resistance points. According to the authors, these findings may be explained by cultural factors.

Berk et al. (2017) examined the prices of 77 individual stocks belonging to 15 frontier equity markets. Overall, psychological barriers were found to be a feature of frontier market equity pricing. A large number of securities exhibited predictable pricing patterns after passing through a psychologically important price point, including round numbers.

On a related vein, Carrera (2015) used the notion of price barrier to assess the exchange rates of 15 Latin American countries. It was found evidence of deviations in the exchange rates relative to the US dollar from a long-run equilibrium perspective.

The current study contributes to the scarce literature on psychological barriers in emerging markets by analyzing the phenomenon in a set of eight Latin American stock market indices.

Most of the literature on psychological barriers consider developed market indices. For example, Donaldson (1990a, 1990b) tested for uniformity in the trailing digits of the Dow Jones Industrial Average (DJIA), the FTSE-100, the TSE, and the Nikkei 225. His findings rejected uniformity for all but the Nikkei index. The results pertaining to the DJIA were later corroborated by Donaldson and Kim (1993) and Ley and Varian (1994).

Koedijk and Stork (1994) expanded the research to the Brussels Stock Index (Belgium), the FAZ General (Germany), the Nikkei 225 (Japan), the S\&P 500 (US), and the FTSE-100 (UK). They discovered significant indications of psychological barriers' existence on the FAZ General, the FTSE-100 and the S\&P 500, but weak indications on the Brussels Index, and none for the Nikkei 225.

De Ceuster, Dhaene and Schatteman (1998) compared the last digits of DJIA, FTSE-100, or the Nikkei 225 with the empirical distribution of a Monte Carlo simulation. They did not find any indication of the existence of psychological barriers on those three indices.

Cyree et al. (1999) showed that the last two digits of the DJIA, the S\&P 500, the Financial Times U.K. Actuaries (London) and the DAX are not equally distributed. Prices next to barriers turn up less frequently than prices in a more distant position.

More recently, Dorfleitner and Klein (2009) focused on the DAX 30, the CAC 40, the FTSE-50 and the DJ EURO STOXX 50 for different periods until 2003. They found fragile traces of psychological barriers in all indices at the 1000-level.

The literature on psychological barriers in stock indices continues to be active nowadays. For example, Shawn and Kalaichelvan (2012) examined five European 
indices (FTSE-100, CAC 40, DAX 30, ATX, SMI). They only found evidence for barriers in the SMI at the 1000-level.

Woodhouse et al. (2016) investigated the existence of barriers in the NASDAQ Composite index from 1971 to 2012. Statistically significant barrier effects were detected in certain index levels (usually in multiples of 100).

Different studies concluded that price barriers or at least significant deviations from uniformity also exist in other asset classes such as exchange rates (De Grauwe and Decupere, 1992; Mitchell and Izan, 2006), bonds (Burke, 2001), commodities (Aggarwal and Lucey, 2007; Lucey and O'Connor, 2016), derivatives (Schwartz, Van Ness and Van Ness, 2004; Chen and Tai, 2011; Jang et al., 2015; Dowling, Cummins and Lucey, 2016; Palao and Pardo, 2018) and cryptocurrencies (Fonseca, Pacheco and Lobão, 2020; Li, li and Chong, 2020). Overall, evidence of price barriers in various asset classes seems to be fairly robust.

\section{DATA AND METHODOLOGY}

\subsection{Data}

All the indices under scrutiny use a weighted market capitalization to measure performance. The examination window for each of the stock market indices under study is presented in Table 1 below. Starting dates are different since we used the data pertaining each index since its inception. The data have daily frequency and were retrieved from Thomson Reuters Datastream and, in the case of the Colombian stock market, from the respective official stock exchange website. Summary statistics on the stock prices are presented in Table 2 where it can be seen that all the markets of the sample had a positive mean return in the sample period. Brazil and Argentina present the most volatile markets in the region. There is no pattern in the asymmetry of the returns distributions. Moreover, all the stock markets show a number of outliers inconsistent with normality. This conclusion is corroborated by the results of the Jarque-Bera test.

\subsection{Methodology}

Regarding existence of positional effects, we investigate if the indices close more or less frequently around round numbers by performing a number of uniformity tests and barrier tests on the M-values of the closing prices, as it will be described in the following sections. The presence of transgressional effects is captured by investigating the dynamics of the conditional mean return and conditional variance before and after the crossing of a barrier. We use regression and GARCH analysis to assess the differential impact of being above or below a possible barrier. 


\section{TABLE 1}

DATA USED IN THE STUDY

\begin{tabular}{|l|c|c|c|}
\hline \multicolumn{1}{|c|}{ Series } & Stock Index & Starting date & Ending date \\
\hline Argentina & MERVAL & October $19^{\text {th }}, 1989$ & \\
Brazil & BOVESPA & January $3^{\text {rd }}, 1994$ & \\
Chile & IPSA & September $27^{\text {th }}, 1987$ & \\
Colombia & COLCAP & January $14^{\text {th }}, 2008$ & \multirow{2}{*}{ December $31^{\text {st }}, 2015$} \\
Mexico & IPC & January 4 4 th 1988 & \\
Panama & BVPSI & January $1^{\text {st }}, 1992$ & \\
Peru & IGBVL & January $2^{\text {nd }}, 1991$ & \\
Venezuela & IBVC & April $1^{\text {st }}, 1993$ & \\
\hline
\end{tabular}

TABLE 2

SUMMARY STATISTICS ON STOCK PRICES DATA SERIES

\begin{tabular}{|l|c|c|c|r|r|r|r|r|}
\hline \multirow{2}{*}{ Series } & & \multicolumn{9}{|c|}{ Obs. } & Mean & \multicolumn{1}{c|}{$\begin{array}{c}\text { Std. } \\
\text { Dev. }\end{array}$} & Skewness & Kurtosis & $\begin{array}{c}\text { Jarque-Bera } \\
\text { stat. } \\
\text { (p-value) }\end{array}$ & Min. & Max. \\
\hline Argentina & 6835 & 0.000930 & 0.027991 & -2.138783 & 90.23957 & 0.00000 & 15.89 & 14173.87 \\
Brazil & 5738 & 0.000825 & 0.022271 & 0.513736 & 14.89715 & 0.00000 & 380.09 & 73516.00 \\
Chile & 5809 & 0.000334 & 0.011119 & 0.237394 & 10.76731 & 0.00000 & 538.01 & 5040.97 \\
Colombia & 2079 & 0.000058 & 0.011059 & -0.633427 & 9.714583 & 0.00000 & 686.64 & 1942.37 \\
Mexico & 7303 & 0.000828 & 0.015262 & 0.068445 & 10.42913 & 0.00000 & 86.61 & 46357.24 \\
Panama & 6261 & 0.000540 & 0.008115 & 5.102059 & 163.41840 & 0.00000 & 13.70 & 478.75 \\
Peru & 6521 & 0.000914 & 0.014736 & -0.076298 & 11.31932 & 0.00000 & 22.92 & 24051.62 \\
Venezuela & 5935 & 0.001651 & 0.017485 & 0.836699 & 21.70214 & 0.00000 & 0.76 & 15580.47 \\
\hline
\end{tabular}

\subsubsection{Definition of Barriers}

Following Brock, Lakonishok and LeBaron (1992) and Dorfleitner and Klein (2009), we will use the so-called band technique and barriers will thus be defined as a certain range around the actual barrier. The main reason is that market participants will most certainly become active at a certain level before the index touches a round price level. Considering an index level of 100, for instance, over-excitement is expected to begin for instance at 99 or 101, or even at 95 or 105. Barriers will thus be defined as multiples of the $l$ th power of ten, with intervals with an absolute length of $2 \%$, $5 \%, 10 \%$, and $25 \%$ of the corresponding power of ten as barriers. These intervals are conventionally used in the literature about psychological barriers. Formally, we may consider possible barrier bands: 
M1000: Barrier level $l=4(10000$ s) $\quad 10,000 \pm 200 ; 10,000 \pm 250 ; 10,000 \pm 500 ; 10,000 \pm 1,000$

M100: Barrier level $l=3(1000 s) \quad 1,000 \pm 20 ; 1,000 \pm 25 ; 1,000 \pm 50 ; 1,000 \pm 100$

M10: Barrier level $l=2(100 \mathrm{~s}) \quad 100 \pm 2 ; 100 \pm 2.5 ; 100 \pm 5 ; 100 \pm 10$

M1: Barrier level $l=1(10 \mathrm{~s}) \quad 10 \pm 0.2 ; 10 \pm 0.25 ; 10 \pm 0.5 ; 10 \pm 1$

M0.1: Barrier level $l=0(1 \mathrm{~s}) \quad 1 \pm 0.02 ; 1 \pm 0.025 ; 1 \pm 0.05 ; 1 \pm 0.1$

\subsubsection{M-Values}

$M$-values refer to the last digits in the integer portion of the indices under analysis. Initially used by Donaldson and Kim (1993), $M$-values consider potential barriers at the levels ..., 300, 400, .., 3400, 3500, i.e. at:

$$
k \times 100, k=1,2, \ldots
$$

Later, De Ceuster, Dhaene and Schatteman (1998) claimed that this definition was too narrow because the series was not multiplicatively regenerative, resulting, for instance, on 3400 being considered a barrier, whereas 340 would not. Additionally, the authors claimed that, as defined by Eq. (1), the gap between barriers would tend to zero as the price series increased, disrupting the intuitive appeal of a psychological barrier. Thus, one should also consider the possibility of barriers at the levels ..., 10, $20, \ldots, 100,200, \ldots, 1000,2000, \ldots$, i.e. at:

$$
k \times 10^{l}, k=1,2, \ldots, 9 ; l=\ldots,-1,0,1, \ldots ;
$$

and, on the other hand, at the levels $\ldots, 10,11, \ldots, 100,110, \ldots, 1000,1100, \ldots$, i.e. at:

$$
k \times 10^{l}, k=10,11, \ldots, 99 ; l=\ldots,-1,0,1, \ldots ;
$$

$M$-values would then be defined according to these barriers. For barriers at the levels defined in Eq. (1), $M$-values would be the pair of digits preceding the decimal point:

$$
M_{t}^{a}=\left[P_{t}\right] \bmod 100
$$

where $P_{t}$ is the integer part of $P_{t}$ and $\bmod 100$ refers to the reduction modulo 100 . For barriers at the levels defined by Eq. (2) and Eq. (3), the $M$-values would be defined respectively as the second and third and the third and fourth significant digits. Formally,

$$
\begin{aligned}
& M_{t}^{b}=\left[100 \times 10^{\left(\log P_{t}\right) \bmod 1}\right] \bmod 100 ; \\
& M_{t}^{c}=\left[1000 \times 10^{\left(\log P_{t}\right) \bmod 1}\right] \bmod 100 ;
\end{aligned}
$$


where logarithms are to base 10. In practical terms, for example if $P_{t}=1234.56$, then $M_{t}^{a}=34$. At this level, barriers should appear when $M_{t}^{a}=00$. Additionally, $M_{t}^{b}=23$ and $M_{t}^{c}=12$.

\subsubsection{Uniformity Test}

Having computed the $M$-values, the next step consists of examining the uniformity of their distribution. Following Aggarwal and Lucey (2007), this will be done through a Kolmogrov-Smirnov Z-statistic test. Thus we will be testing H0: uniformity of the M-values distribution against $\mathrm{H} 1$ : non-uniformity of the M-values distribution.

It is important to emphasize that the rejection of uniformity might suggest the existence of significant psychological barriers but it is not in itself sufficient to prove their existence. Ley and Varian (1994) showed that the last digits of the Dow Jones Industrial Average were in fact not uniformly distributed and even appeared to exhibit certain patterns, but the returns conditional on the digit realization were still significantly random. Additionally, De Ceuster, Dhaene and Schatteman (1998) noted that as a series grows without limit and the intervals between barriers become wider, the theoretical distribution of digits and the respective frequency of occurrence is no longer uniform.

\subsubsection{Barrier Tests}

Barrier tests are used to assess whether observations are less frequent near barriers than it would be expected considering a uniform distribution. The existence of a psychological barrier implies we will observe a significantly lower closing price frequency within an interval around the barrier (Donald and Kim, 1993; Ley and Varian, 1994). Therefore, the objective of the barrier tests is to investigate the influence of round numbers in the non-uniform distribution of $M$-values. We will use two types of barrier tests: the barrier proximity test and the barrier hump test.

\section{a) Barrier Proximity Test}

This test examines the frequency of observations, $\mathrm{f}(\mathrm{M})$, near potential barriers and will be performed according to Eq. (7).

$$
f(M)=\alpha+\beta D+\varepsilon
$$

The dummy variable will take the value of unity when the index is at the supposed barrier and zero elsewhere. As it was mentioned in section 3.2.1, this barrier will not be strictly considered as an exact number but also as a number of different specific intervals, namely with an absolute length of 2\%, 5\%, $10 \%$ and $25 \%$ of the corresponding 
power of ten as barriers. The null hypothesis of no barriers will thus imply that $\beta$ equals zero, while $\beta$ is expected to be negative and significant in the presence of barriers as a result of lower frequency of $M$-values at these levels.

\section{b) Barrier Hump Test}

The second barrier test will examine not just the tails of frequency distribution near the potential barriers, but the entire shape of the distribution. It is thus necessary to define the alternative shape that the distribution should have in the presence of barriers (Donaldson and Kim, 1993; Aggarwal and Lucey, 2007). Bertola and Caballero (1992), who analysed the behaviour of exchange rates in the presence of target zones imposed by forward-looking agents, suggest that a hump-shape is an appropriate alternative for the distribution of observations.

The test to examine this possibility will follow Eq. (8), in which the frequency of observation of each $M$-value is regressed on the $M$-value itself and on its square.

$$
f(M)=\alpha+\Phi M+\gamma M^{2}+\eta
$$

Under the null hypothesis of no barriers $Y$ is expected to be zero, whereas the presence of barriers should result in $\Upsilon$ being negative and significant.

\subsubsection{Conditional Effect Tests}

The study of positional effects should to be complemented with the investigation regarding transgressional effects that result from psychological barriers (Ley and Varian, 1994). Therefore, it is necessary to analyse the dynamics of the returns series around these barriers, namely regarding mean and variance in order to examine the differential effect on returns due to prices being near a barrier, and whether these barriers were being approached on an upward or on a downward movement (Cyree et al., 1999; Aggarwal and Lucey, 2007).

Accordingly, we will thus define four regimes around barriers: BD for the five days before prices reaching a barrier on a downward movement, $\mathrm{AD}$ for the five days after prices crossing a barrier on a downward movement, and BU and AU for the five days respectively before and after prices breaching a barrier on an upward movement. These dummy variables will take the value of unity for the days noted and zero otherwise. To examine the robustness of the results to the assumption that the regime should last for five days, we will also consider a window of ten days. In the absence of barriers, we expect the coefficients on the indicator variables in the mean equation to be non-significantly different from zero.

$$
R_{t}=\beta_{1}+\beta_{2} B D_{t}+\beta_{3} A D_{t}+\beta_{4} B U_{t}+\beta_{5} A U_{t}+\varepsilon_{t}
$$


Following Aggarwal and Lucey (2007), we started with an OLS estimation of Eq. (9) but heteroscedasticity and autocorrelation were present across our data base. Therefore, the full analysis of the effects in the proximity of barriers required us to apply the former test also to the variances. Eq. (10) represents this approach assuming autocorrelation similar to one as in Cyree et al. (1999) and Aggarwal and Lucey (2007). Besides the abovementioned dummy variables it includes a moving average parameter and a GARCH parameter.

$$
\begin{gathered}
\varepsilon_{t}=N\left(0, V_{t}\right) \\
V_{t}=\alpha_{1}+\alpha_{2} B D_{t}+\alpha_{3} A D_{t}+\alpha_{4} B U_{t}+\alpha_{5} A U_{t}+\alpha_{6} V_{\mathrm{t}-1}+\alpha_{7} \varepsilon_{t-1}^{2}+\eta_{t}
\end{gathered}
$$

The four possible hypothesis to be tested are the following:

H1: There is no difference in the conditional mean return before and after a downward crossing of a barrier.

$\mathrm{H} 2$ : There is no difference in the conditional mean return before and after an upward crossing of a barrier.

H3: There is no difference in conditional variance before and after a downward crossing of a barrier.

H4: There is no difference in the conditional variance before and after a upward crossing of a barrier.

\section{EMPIRICAL FINDINGS}

\subsection{Uniformity Test}

Table 3 provides the results of a uniformity test concerning the distribution of digits for the eight stock market indices under scrutiny. Overall, there is robust evidence that the $M$-values do not follow a uniform distribution in each one of the stock markets included in the sample. Moreover, uniformity is more clearly rejected in in the highest barrier levels. In the case of the markets of Panama and Peru, uniformity is rejected at $1 \%$ in all the barrier levels. The rejection of uniformity of the trailing digits is not so strong in the Colombian market: from the four barrier levels under test, uniformity is rejected at a significance level of 5\% only in the two highest barrier levels. 
TABLE 3

KOLMOGOROV-SMIRNOV TEST FOR UNIFORMITY OF DIGITS

\begin{tabular}{|l|c|c|c|c|c|}
\hline Series & M0.1 $(l=0)$ & M1 $(l=1)$ & M10 $(l=2)$ & M100 $(l=3)$ & M1000 $(l=4)$ \\
\hline Argentina & 1.2843 & 1.5080 & 2.5877 & 8.5761 & 9.8682 \\
& $(0.0738)^{*}$ & $(0.0212)^{* *}$ & $(0.0000)^{* * *}$ & $(0.0000)^{* * *}$ & $(0.0000)^{* * *}$ \\
Brazil & 1.1264 & 5.0241 & 1.3029 & 7.4010 & 75.6622 \\
& $(0.1581)$ & $(0.0000)^{* * *}$ & $(0.0671)^{*}$ & $(0.0000)^{* * *}$ & $(0.0000)^{* * *}$ \\
Chile & 1.5140 & 1.1853 & 2.1372 & 17.3372 & - \\
& $(0.0204)^{* *}$ & $(0.1204)$ & $(0.0002)^{* * *}$ & $(0.0000)^{* * *}$ & \\
Colombia & 0.8746 & 1.1847 & 1.4204 & 8.2443 & - \\
& $(0.4288)$ & $(0.1207)$ & $(0.0354)^{* *}$ & $(0.0000)^{* * *}$ & \\
Mexico & 1.1184 & 2.1404 & 1.1626 & 8.1189 & 10.1611 \\
& $(0.1638)$ & $(0.0002)^{* * *}$ & $(0.1339)$ & $(0.0000)^{* * *}$ & $(0.0000)^{* * *}$ \\
Panama & 2.8199 & 6.4622 & 17.9263 & - & - \\
& $(0.0000)^{* * *}$ & $(0.0000)^{* * *}$ & $(0.0000)^{* * *}$ & & \\
Peru & 1.9372 & 2.1206 & 2.4100 & 10.3814 & - \\
& $(0.0011)^{* * *}$ & $(0.0002)^{* * *}$ & $(0.0000)^{* * *}$ & $(0.0000)^{* * *}$ & \\
Venezuela & 1.7060 & 6.9328 & 1.2623 & 1.9841 & 6.2357 \\
& $(0.0059)^{* * *}$ & $(0.0000)^{* * *}$ & $(0.0826)^{*}$ & $(0.0008)^{* * *}$ & $(0.0000)^{* * *}$ \\
\hline
\end{tabular}

Table 3 shows the results of a Kolmogorov-Smirnov test for uniformity. Each test was performed for the daily closing prices of each stock index. P-values are in parenthesis. H0: uniformity in the distribution of digits, H1: non uniformity in the distribution of digits. ***: significant at the 1 percent level; **: significant at the 5 percent level; *: significant at the 10 percent level.

\subsection{Barrier Tests}

\subsubsection{Barrier Proximity Test}

Results for the barrier proximity tests are shown in Tables 4 to 8 for the intervals mentioned in sections 3.2.1 and 3.2.4. As referred above, in the presence of a barrier we would expect $\beta$ to be negative and significant, implying a lower frequency of $M$-values at these points. Considering a barrier in the exact zero module point, evidence in Table 4 shows that only Brazil at the 10-level barrier seem to reject the no barrier hypothesis at a statistical significance of $10 \%$. If we assume a barrier to be in the interval 98-02, there are four markets that seem to reject the no barrier hypothesis at a statistical significance of 5\%: Panama at the lower barrier level and Mexico, Peru, and Venezuela at the 1000-level barrier. As we keep widening the barrier interval, evidence appear to confirm the results for three of these four countries. In fact, considering the 95-05 interval, Table 6 shows that the no barrier hypothesis is again rejected for Panama, Mexico and Peru at the same barrier levels. The difference is that the results for Mexico and Peru are now statistically significant at the $1 \%$ level. In addition, Moreover, Table 6 shows that in the case of the Argentinian and Colombian markets the no barrier hypothesis is rejected at the 1000-level barrier at a statistical 


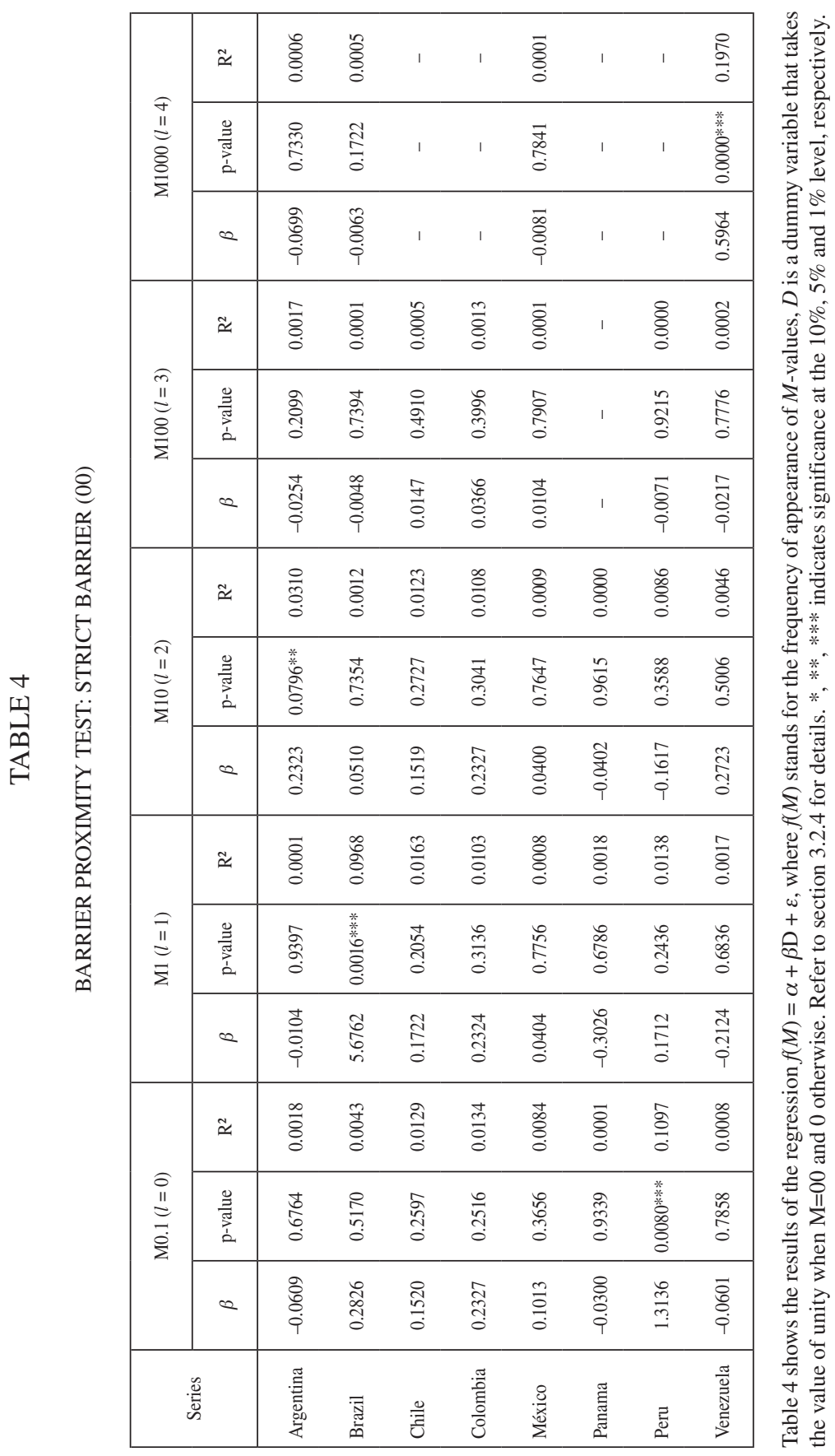




\begin{tabular}{|c|c|c|c|c|c|c|c|c|c|c|c|}
\hline \multirow{16}{*}{ 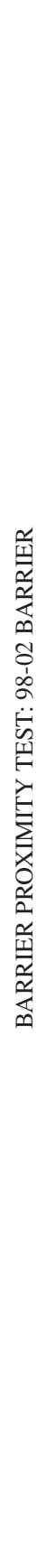 } & \multirow{3}{*}{ 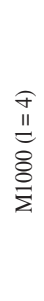 } & $\approx$ & ¿̊̊. & छ̊े & 1 & 1 & 号 & 1 & 1 & : & 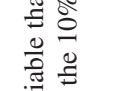 \\
\hline & & 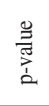 & ๙ૂ. & స్ర్లి & 1 & 1 & 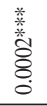 & 1 & 1 & 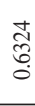 & 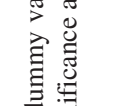 \\
\hline & & $\infty$ & 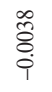 & 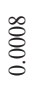 & 1 & 1 & $\begin{array}{l}\infty \\
\stackrel{\circ}{0} \\
\stackrel{0}{0}\end{array}$ & 1 & 1 & 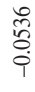 & $\begin{array}{ll}\pi & 50 \\
\infty & 0 \\
0 & 0 \\
0\end{array}$ \\
\hline & & $\approx$ & $\frac{m}{8}$ & $\begin{array}{l}8 \\
8 \\
0 \\
0\end{array}$ & $\frac{+}{\circ}$ & हू. & مُ & 1 & ठั & $\begin{array}{l}\text { oे } \\
\text { ठे } \\
0\end{array}$ & 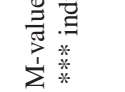 \\
\hline & $\begin{array}{l}\hat{0} \\
\stackrel{11}{=} \\
\frac{8}{2} \\
\frac{1}{2}\end{array}$ & 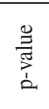 & 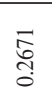 & $\begin{array}{l}\overline{0} \\
\stackrel{0}{0} \\
0\end{array}$ & 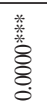 & 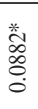 & 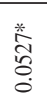 & 1 & $\begin{array}{l}\frac{.}{0} \\
\frac{0}{0} \\
0 \\
0\end{array}$ & 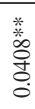 & 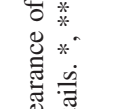 \\
\hline & & $\infty$ & $\frac{\stackrel{n}{0}}{\stackrel{0}{0}}$ & $\begin{array}{l}\text { ôे } \\
\text { †े }\end{array}$ & 茓 & 嵩 & 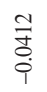 & 1 & $\begin{array}{l}\text { E } \\
\text { S. } \\
\stackrel{0}{0}\end{array}$ & $\begin{array}{l}0 \\
\text { o } \\
\stackrel{0}{0} \\
\stackrel{1}{0}\end{array}$ & 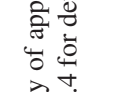 \\
\hline & & $\approx$ & $\frac{n}{3}$ & 命 & 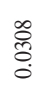 & $\begin{array}{l}8 \\
\vdots \\
0\end{array}$ & ڤ్ & 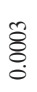 & $\begin{array}{l}\stackrel{\circ}{0} \\
\stackrel{0}{0}\end{array}$ & ठ̊. & 窇 \\
\hline & $\begin{array}{l}\frac{1}{11} \\
\stackrel{2}{\Xi} \\
\stackrel{0}{\Sigma}\end{array}$ & 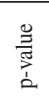 & तิ & $\begin{array}{l}\tilde{n} \\
\text { ó } \\
0\end{array}$ & $\begin{array}{l}\text { 苂 } \\
\stackrel{0}{\circ} \\
\stackrel{0}{0} \\
0\end{array}$ & \begin{tabular}{l}
$\infty$ \\
\multirow{2}{0}{} \\
s.
\end{tabular} & $\stackrel{\infty}{\Xi}$ & \begin{tabular}{l}
\multirow{2}{0}{} \\
$\stackrel{0}{0}$ \\
0 \\
0
\end{tabular} & ๙̊. & गु & 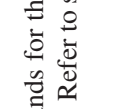 \\
\hline & & $\infty$ & $\begin{array}{l}\tilde{0} \\
\tilde{0} \\
0\end{array}$ & लि. & $\frac{\hat{O}}{0}$ & $\begin{array}{l}\infty \\
\tilde{o}_{0} \\
\grave{i}\end{array}$ & $\begin{array}{l}\text { to } \\
\text { 。 } \\
\text { : }\end{array}$ & $\begin{array}{l}\text { t. } \\
\text { ठ } \\
0\end{array}$ & $\stackrel{\infty}{\stackrel{0}{0}}$ & $\frac{N}{\stackrel{2}{\circ}}$ & $\sum_{t=}^{\infty} \sum_{D=0}^{\infty}$ \\
\hline & & $\approx$ & 三 & 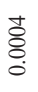 & ठ̊̊ & 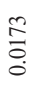 & 离 & ๙े. & ஜ̊ & $\stackrel{0}{\tilde{\delta}}$ & 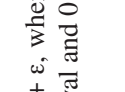 \\
\hline & $\begin{array}{l}\stackrel{=}{\text { II }} \\
\stackrel{=}{\Sigma}\end{array}$ & 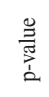 & $\begin{array}{l}\text { ले } \\
\text { ஸे } \\
0\end{array}$ & $\begin{array}{l}\text { o } \\
\text { oे } \\
\text { o. } \\
0\end{array}$ & $\begin{array}{l}\text { के } \\
\infty \\
\infty \\
0 \\
0\end{array}$ & $\begin{array}{l}\frac{1}{0} \\
\frac{1}{0}\end{array}$ & $\begin{array}{l}n \\
\substack{f \\
\infty \\
0 \\
0}\end{array}$ & 交 & 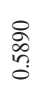 & ळે & 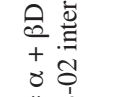 \\
\hline & & $\infty$ & है & $\begin{array}{l}\text { E } \\
\text { : } \\
1\end{array}$ & $\begin{array}{l}\stackrel{\circ}{\circ} \\
\stackrel{0}{0}\end{array}$ & 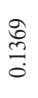 & $\begin{array}{l}\stackrel{0}{1} \\
\stackrel{0}{0} \\
\stackrel{1}{1}\end{array}$ & 拿 & $\begin{array}{l}\tilde{\delta} \\
\stackrel{0}{0} \\
\stackrel{1}{1}\end{array}$ & 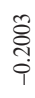 & $\underset{\underbrace{}}{\sum_{0} \underset{\Xi}{\Xi}}$ \\
\hline & & $\approx$ & $\stackrel{n}{\circ}$ & $\bar{\Xi}$ & : & 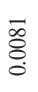 & ๕ి & $\begin{array}{l}\overline{\text { ల్ }} \\
\dot{0}\end{array}$ & $\stackrel{n}{a}$ & F्ञ & 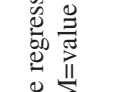 \\
\hline & $\begin{array}{l}0 \\
\text { II } \\
= \\
0\end{array}$ & 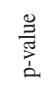 & 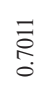 & 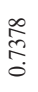 & \begin{tabular}{l}
$\infty$ \\
\multirow{0}{+}{} \\
0 \\
0
\end{tabular} & 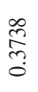 & $\begin{array}{l}\stackrel{0}{0} \\
\cdots \\
0\end{array}$ & $\begin{array}{l}\text { 今. } \\
\text { 今े } \\
\text { ¿. }\end{array}$ & ڤ్ & 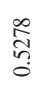 & 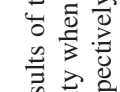 \\
\hline & & $\infty$ & 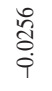 & $\begin{array}{l}\text { 委 } \\
\text { क्ष }\end{array}$ & 咅 & $\begin{array}{l}\text { In } \\
\text { o. } \\
0 \\
0\end{array}$ & $\begin{array}{l}\text { ڤे } \\
\text { ठे }\end{array}$ & $\begin{array}{l}\text { خे } \\
\text { ஸे } \\
\text { iे }\end{array}$ & तె & $\begin{array}{l}\hat{\delta} \\
0 \\
0\end{array}$ & 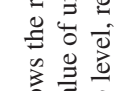 \\
\hline & & & $\begin{array}{l}\text { 穿 } \\
\text { 总 } \\
\text { 妾 }\end{array}$ & 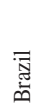 & 苛 & 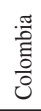 & 总 & 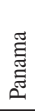 & ᄅ. & 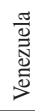 & 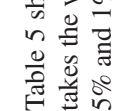 \\
\hline
\end{tabular}




\begin{tabular}{|c|c|c|c|c|c|c|c|c|c|c|c|}
\hline \multirow{16}{*}{ 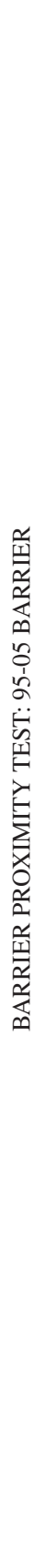 } & \multirow{3}{*}{ 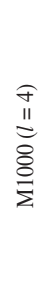 } & $\approx$ & $\overline{\widehat{\Xi}}$ & 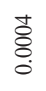 & 1 & 1 & ¿̊̊. & 1 & 1 & ఫ్ & \multirow{4}{*}{ 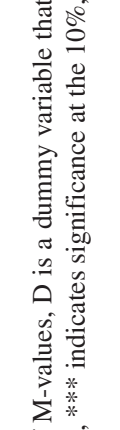 } \\
\hline & & 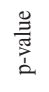 & 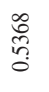 & 売 & 1 & 1 & $\frac{\infty}{\infty}$ & 1 & 1 & हे हे & \\
\hline & & $\infty$ & 灾 & 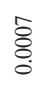 & 1 & 1 & ठ̊̊. & 1 & 1 & $\begin{array}{l}\text { 某 } \\
\text { 它 } \\
\stackrel{9}{i}\end{array}$ & \\
\hline & \multirow{3}{*}{ 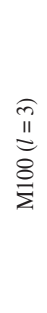 } & $\approx$ & \%ิ & ठ̊̊̊ & $\begin{array}{l}\text { ते } \\
\text { तิ }\end{array}$ & 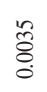 & $\frac{\sigma}{0}$ & I & $\stackrel{n}{\circ}$ & 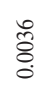 & \\
\hline & & 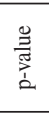 & $\begin{array}{l}\text { 苑 } \\
\stackrel{\circ}{0} \\
\stackrel{0}{0}\end{array}$ & 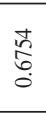 & ठ̊̊. & $\frac{9}{0}$ & $\begin{array}{l}\text { 姜 } \\
\frac{8}{8} \\
0 \\
\end{array}$ & 1 & 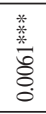 & $\frac{\mathscr{m}}{\stackrel{n}{0}}$ & 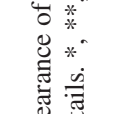 \\
\hline & & $\infty$ & $\begin{array}{l}\frac{n}{a} \\
\stackrel{0}{0} \\
\stackrel{9}{0}\end{array}$ & 灾 & 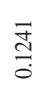 & 离 & 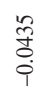 & I & 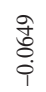 & 홍 & \\
\hline & \multirow{3}{*}{$\begin{array}{l}\text { I } \\
\text { II } \\
\stackrel{0}{0} \\
\stackrel{\Sigma}{\Sigma}\end{array}$} & $\approx$ & $\begin{array}{l}\stackrel{0}{0} \\
\overparen{0} \\
0 \\
0\end{array}$ & 志 & $\begin{array}{l}\text { ते } \\
\text { ठे. }\end{array}$ & $\begin{array}{l}\text { \& } \\
\stackrel{0}{0} \\
0\end{array}$ & 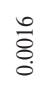 & $\begin{array}{l}\frac{n}{8} \\
0 \\
0\end{array}$ & 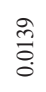 & ठิ & 苛. \\
\hline & & 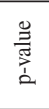 & $\frac{\infty}{0}$ & ్ㅗㅇ & $\stackrel{*}{\stackrel{*}{0}}$ & $\begin{array}{l}\overrightarrow{0} \\
\text { o. } \\
0\end{array}$ & مِّ & 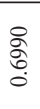 & 竎 & ڤิ & $\begin{array}{ll}\overrightarrow{0} \\
\vec{d} \\
\vec{d}\end{array}$ \\
\hline & & $\infty$ & $\begin{array}{l}\text { 总 } \\
\stackrel{0}{0} \\
0\end{array}$ & $\begin{array}{l}\text { त్ } \\
\text { ठ. }\end{array}$ & $\begin{array}{l}\overline{\widehat{\sigma}} \\
\dot{0}\end{array}$ & $\begin{array}{l}\infty \\
\stackrel{\delta}{0} \\
0 \\
0\end{array}$ & $\frac{0}{0}$ & $\begin{array}{l}\text { J } \\
\frac{0}{0}\end{array}$ & $\begin{array}{l}2 \\
\stackrel{8}{8} \\
\stackrel{9}{9}\end{array}$ & 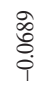 & 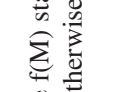 \\
\hline & \multirow{3}{*}{$\begin{array}{l}\widehat{I} \\
\stackrel{\Xi}{\Xi} \\
\vec{\Sigma}\end{array}$} & $\approx$ & $\begin{array}{l}\infty \\
\text { ల్ర } \\
\stackrel{0}{0}\end{array}$ & 灾 & ठ્ठે & $\begin{array}{l}\text { D. } \\
\stackrel{0}{0} \\
0\end{array}$ & ¿̊̊. & $\begin{array}{l}\infty \\
\stackrel{0}{0} \\
\dot{0}\end{array}$ & 灾 & $\stackrel{\infty}{8}$ & \multirow{7}{*}{ 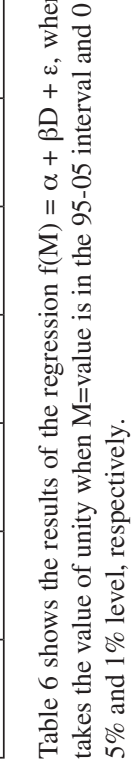 } \\
\hline & & 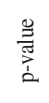 & $\begin{array}{l}\stackrel{*}{*} \\
\stackrel{*}{0} \\
\stackrel{0}{0}\end{array}$ & $\frac{\infty}{\infty}$ & 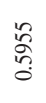 & 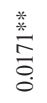 & $\begin{array}{l}\stackrel{\circ}{\circ} \\
\stackrel{\circ}{0}\end{array}$ & $\begin{array}{l}\overline{7} \\
\text { ț } \\
\text { of }\end{array}$ & 亲 & है & \\
\hline & & $\infty$ & مิ & $\begin{array}{l}\text { 今े } \\
\text { o. } \\
\text { i }\end{array}$ & 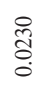 & 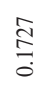 & $\begin{array}{l}\circ \\
\stackrel{8}{8} \\
\stackrel{0}{0}\end{array}$ & $\frac{\stackrel{n}{g}}{\dot{q}}$ & ڤి & $\begin{array}{l}\circ \\
\stackrel{8}{0} \\
\stackrel{0}{0}\end{array}$ & \\
\hline & \multirow{3}{*}{ 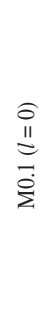 } & $\approx$ & ठ̊ & 蒙 & 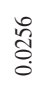 & 守 & 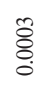 & 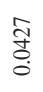 & 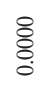 & ᄋ̊̊. & \\
\hline & & 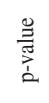 & \begin{tabular}{l}
5 \\
\multirow{2}{5}{} \\
0
\end{tabular} & $\begin{array}{l}\text { హ్ } \\
\text { రి }\end{array}$ & 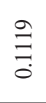 & त్ & $\underset{\substack{\infty \\
0}}{\overrightarrow{0}}$ & 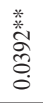 & 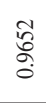 & $\begin{array}{l}20 \\
0 \\
\infty \\
0 \\
0\end{array}$ & \\
\hline & & $\infty$ & $\begin{array}{l}+ \\
\stackrel{8}{\circ} \\
\stackrel{+}{\circ}\end{array}$ & $\begin{array}{l}\vec{n} \\
\text { 号 } \\
0\end{array}$ & $\begin{array}{l}\stackrel{0}{\circ} \\
\stackrel{8}{0} \\
0\end{array}$ & $\begin{array}{l}\vec{\infty} \\
\stackrel{5}{0} \\
\dot{0}\end{array}$ & 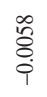 & 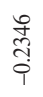 & 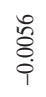 & $\begin{array}{l}\stackrel{\Xi}{a} \\
\stackrel{0}{\oplus}\end{array}$ & \\
\hline & \multicolumn{2}{|c|}{ 怨 } & 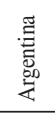 & 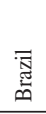 & 苛 & हू. & 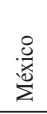 & 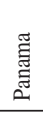 & 壳 & 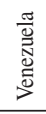 & \\
\hline
\end{tabular}




\begin{tabular}{|c|c|c|c|c|c|c|c|c|c|c|c|}
\hline \multirow{16}{*}{ 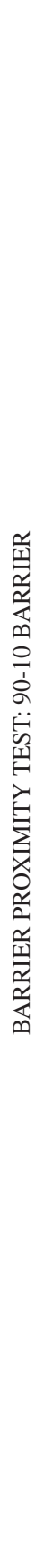 } & \multirow{3}{*}{ 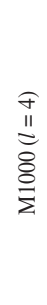 } & $\approx$ & 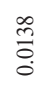 & ठ̊. & 1 & 1 & 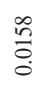 & 1 & 1 & $\begin{array}{l}\text { ర్ర } \\
\stackrel{0}{0}\end{array}$ & \multirow{4}{*}{ 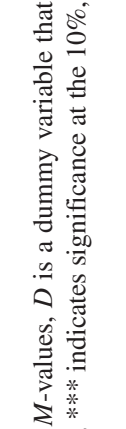 } \\
\hline & & 总 & $\begin{array}{l}\stackrel{\circ}{\circ} \\
\text { o }\end{array}$ & 晜 & 1 & 1 & 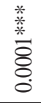 & 1 & 1 & 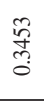 & \\
\hline & & $\infty$ & 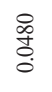 & 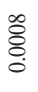 & 1 & 1 & 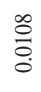 & 1 & 1 & 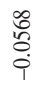 & \\
\hline & & $\approx$ & $\begin{array}{l}\infty \\
\stackrel{0}{0} \\
0\end{array}$ & 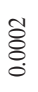 & 命 & 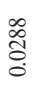 & స్ ठ & 1 & $\begin{array}{l}\text { ळ్ } \\
\text { o. }\end{array}$ & $\begin{array}{l}\text { ठे } \\
\text { ठ }\end{array}$ & \\
\hline & $\begin{array}{l}\text { II } \\
\text { II } \\
\frac{8}{2} \\
\Sigma\end{array}$ & 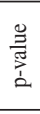 & 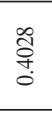 & 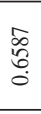 & $\begin{array}{l}\text { 蕫 } \\
8 \\
8 \\
0\end{array}$ & 蕫 & 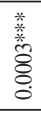 & 1 & 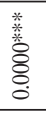 & 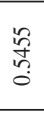 & 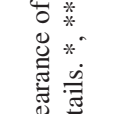 \\
\hline & & $\infty$ & 范 & 产 & $\begin{array}{l}\text { Oू } \\
\stackrel{0}{0} \\
0\end{array}$ & $\begin{array}{l}\text { to } \\
\text { +े. } \\
\text { i }\end{array}$ & $\begin{array}{l}\vec{\infty} \\
\text { oे } \\
\stackrel{i}{i}\end{array}$ & I & $\frac{8}{\stackrel{1}{0}}$ & $\stackrel{\infty}{\Xi}$ & 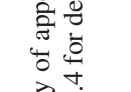 \\
\hline & \multirow{3}{*}{$\begin{array}{l}\text { त } \\
\text { II } \\
\vdots \\
\Sigma \\
\Sigma\end{array}$} & $\approx$ & $\begin{array}{l}\stackrel{\infty}{\widetilde{J}} \\
\stackrel{0}{0}\end{array}$ & 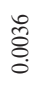 & $\frac{\text { fo }}{8}$ & \begin{tabular}{l} 
ஜू \\
\hdashline \\
0
\end{tabular} & $\begin{array}{l}8 \\
8 \\
0\end{array}$ & ठิ̊ & $\begin{array}{l}\text { సे } \\
\text { ठे }\end{array}$ & $\begin{array}{l}\overline{0} \\
\stackrel{0}{0}\end{array}$ & 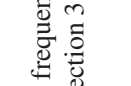 \\
\hline & & 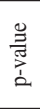 & $\stackrel{\widetilde{I}}{\stackrel{\widetilde{J}}{0}}$ & 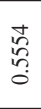 & 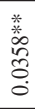 & $\begin{array}{c}\text { f } \\
\stackrel{\text { f }}{\delta}\end{array}$ & $\begin{array}{l}\circ \\
\vdots \\
\sigma \\
0\end{array}$ & 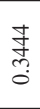 & $\begin{array}{l}\text { \% } \\
\text { Oे } \\
0 \\
0\end{array}$ & 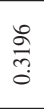 & 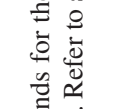 \\
\hline & & $\infty$ & $\begin{array}{l}\text { ?̊ } \\
\text { ț. }\end{array}$ & & $\begin{array}{l}\text { 总 } \\
\text { - }\end{array}$ & 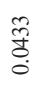 & $\bar{\delta}$ & $\frac{a}{0}$ & $\stackrel{?}{\stackrel{E}{5}}$ & $\begin{array}{l}\widetilde{\alpha} \\
\text { oे } \\
\text { Oें }\end{array}$ & $\sum_{0}^{\infty}$ \\
\hline & \multirow{3}{*}{$\begin{array}{l}\text { II } \\
\stackrel{=}{\Xi} \\
\text { ह }\end{array}$} & $\approx$ & $\begin{array}{l}\tilde{n} \\
\tilde{o} \\
0\end{array}$ & 苂 & $\begin{array}{l}\overline{8} \\
8 \\
0\end{array}$ & $\begin{array}{l}\text { 总 } \\
\text { : }\end{array}$ & 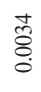 & 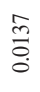 & $\stackrel{n}{\circ}$ & $\begin{array}{l}\tilde{o} \\
\text { o. }\end{array}$ & 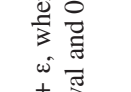 \\
\hline & & 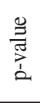 & 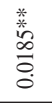 & 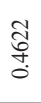 & $\frac{\partial}{\hat{a}}$ & $\frac{m}{m}$ & 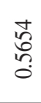 & $\begin{array}{l}\text { of } \\
\text { and } \\
\text { d }\end{array}$ & 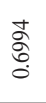 & 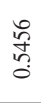 & \\
\hline & & $\approx$ & $\begin{array}{l}0 \\
\stackrel{0}{0} \\
0 \\
0\end{array}$ & 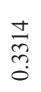 & $\begin{array}{l}\text { वे } \\
\stackrel{0}{0}\end{array}$ & 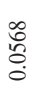 & $\frac{\partial}{\partial}$ & 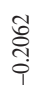 & $\stackrel{\text { ले }}{\stackrel{0}{0}}$ & $\begin{array}{l}\text { oे } \\
\text { : } \\
\text { i. }\end{array}$ & $\underset{\Xi}{\infty}$ \\
\hline & \multirow{3}{*}{ 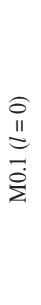 } & $\approx$ & 茴 & $\frac{\circ}{\circ}$ & $\frac{\text { E }}{\stackrel{0}{\circ}}$ & 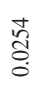 & ळิ & $\begin{array}{l}\bar{m} \\
\text { }\end{array}$ & 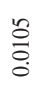 & চ̊̊̊ & \\
\hline & & 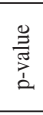 & $\begin{array}{l}\text { Iิ } \\
\text { ปे }\end{array}$ & $\stackrel{\vec{\Xi}}{0}$ & $\frac{\widetilde{\Omega}}{0}$ & $\stackrel{\stackrel{ \pm}{=}}{=}$ & 离 & 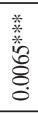 & 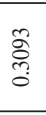 & ふิ & 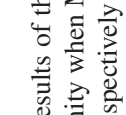 \\
\hline & & $\approx$ & $\begin{array}{l}\text { \&̊ } \\
\stackrel{0}{0} \\
0\end{array}$ & 号 & $\begin{array}{l}\text { वे } \\
\text { Tे } \\
0\end{array}$ & $\begin{array}{l}\text { के } \\
\stackrel{0}{5} \\
0\end{array}$ & 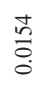 & $\begin{array}{l}\text { हे } \\
\text { ते } \\
\text { के }\end{array}$ & $\begin{array}{l}\text { aे } \\
\text { ô }\end{array}$ & \begin{tabular}{l}
$\infty$ \\
\multirow{8}{0}{} \\
$\stackrel{0}{0}$
\end{tabular} & 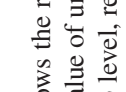 \\
\hline & \multicolumn{2}{|c|}{ 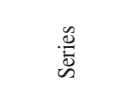 } & 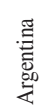 & 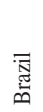 & $\frac{0}{\tilde{z}}$ & 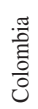 & $\begin{array}{l}\frac{8}{2} \\
\frac{0}{2} \\
\frac{0}{2}\end{array}$ & 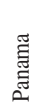 & مّ & 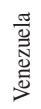 & 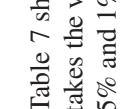 \\
\hline
\end{tabular}




\begin{tabular}{|c|c|c|c|c|c|c|c|c|c|c|c|}
\hline \multirow{16}{*}{ 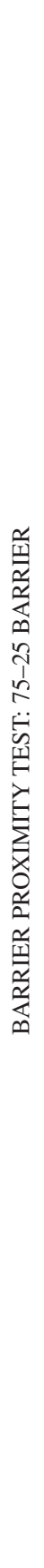 } & \multirow{3}{*}{ 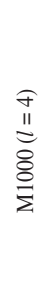 } & $\approx$ & $\overline{\widehat{\Xi}}$ & 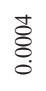 & 1 & 1 & ¿̊̊. & 1 & 1 & ఫ్ & \multirow{4}{*}{ 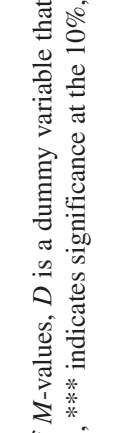 } \\
\hline & & 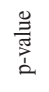 & 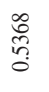 & 売 & 1 & 1 & $\frac{\infty}{\infty}$ & 1 & 1 & हे हे & \\
\hline & & $\infty$ & 灾 & 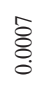 & 1 & 1 & ठ̊̊. & 1 & 1 & $\begin{array}{l}\text { 某 } \\
\text { 它 } \\
\stackrel{9}{i}\end{array}$ & \\
\hline & \multirow{3}{*}{ 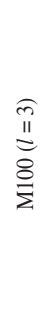 } & $\approx$ & \%ิ & ठ̊̊̊ & $\begin{array}{l}\text { ते } \\
\text { तิ }\end{array}$ & 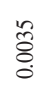 & $\begin{array}{l}5 \\
0 \\
0\end{array}$ & I & $\stackrel{n}{\circ}$ & 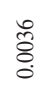 & \\
\hline & & 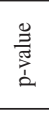 & $\begin{array}{l}\text { 苑 } \\
\stackrel{\circ}{0} \\
\stackrel{0}{0}\end{array}$ & 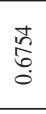 & 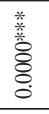 & $\frac{\sigma}{\overrightarrow{0}}$ & $\begin{array}{l}\text { 姜 } \\
\frac{8}{8} \\
0 \\
\end{array}$ & 1 & 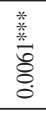 & $\frac{\mathscr{m}}{\stackrel{n}{0}}$ & 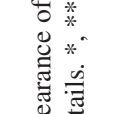 \\
\hline & & $\infty$ & $\begin{array}{l}\frac{n}{a} \\
\stackrel{0}{0} \\
\stackrel{9}{0}\end{array}$ & 灾 & 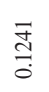 & $\begin{array}{l}\overline{\bar{S}} \\
\stackrel{0}{0}\end{array}$ & 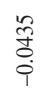 & I & 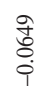 & 홍 & $\begin{array}{l}\text { है } \\
\frac{1}{0}\end{array}$ \\
\hline & \multirow{3}{*}{ 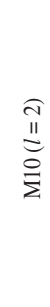 } & $\approx$ & 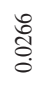 & 志 & ڤે & $\begin{array}{l}\text { \& } \\
\stackrel{0}{0} \\
0\end{array}$ & 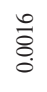 & $\begin{array}{l}\frac{n}{8} \\
0 \\
0\end{array}$ & $\stackrel{\text { के }}{0}$ & ठิ & 胥 \\
\hline & & $\begin{array}{l}\frac{9}{3} \\
\frac{\pi}{2} \\
2\end{array}$ & $\frac{\infty}{0}$ & 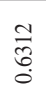 & $\stackrel{*}{\stackrel{*}{0}}$ & $\begin{array}{l}\overrightarrow{0} \\
\text { o. } \\
0\end{array}$ & مِّ & 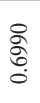 & 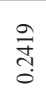 & ڤิ & 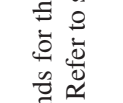 \\
\hline & & $\infty$ & $\begin{array}{l}\text { 总 } \\
\stackrel{0}{0} \\
0\end{array}$ & त्ञ్రి & $\begin{array}{l}\overline{\widehat{\sigma}} \\
\dot{0}\end{array}$ & $\begin{array}{l}\infty \\
\stackrel{\delta}{0} \\
0 \\
0\end{array}$ & $\frac{0}{0}$ & $\begin{array}{l}\text { J } \\
\frac{0}{0}\end{array}$ & $\begin{array}{l}2 \\
8 \\
8 \\
0\end{array}$ & 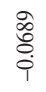 & $\sum_{\substack{\infty \\
0}}^{\infty}$ \\
\hline & \multirow{3}{*}{$\begin{array}{l}\widehat{\bar{I}} \\
\stackrel{ \pm}{=} \\
\bar{\Sigma}\end{array}$} & $\approx$ & $\begin{array}{l}\infty \\
\text { ల్ర } \\
\stackrel{0}{0}\end{array}$ & 灾 & ठ્ठે & $\begin{array}{l}\text { D. } \\
\stackrel{\circ}{0} \\
0 \\
0\end{array}$ & ¿̊̊. & $\begin{array}{l}\infty \\
\check{\delta} \\
0\end{array}$ & 灾 & $\stackrel{\infty}{8}$ & $\begin{array}{l}\frac{5}{3} \\
\omega \\
\omega\end{array}$ \\
\hline & & 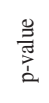 & 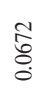 & $\stackrel{\infty}{\stackrel{\infty}{\circ}}$ & $\begin{array}{l}\text { مू } \\
\text { ñ. }\end{array}$ & & $\begin{array}{l}\stackrel{\circ}{\circ} \\
\stackrel{\circ}{0}\end{array}$ & $\begin{array}{l}\overrightarrow{7} \\
\text { ț } \\
\text { on }\end{array}$ & 형 & है & \begin{tabular}{l} 
\pm \\
\multirow{1}{1}{} \\
$i$
\end{tabular} \\
\hline & & $\infty$ & مิ & $\begin{array}{l}\text { 今े } \\
\text { o. } \\
\text { i }\end{array}$ & ্ֻণి & 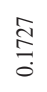 & $\begin{array}{l}\circ \\
\stackrel{8}{8} \\
\stackrel{0}{0}\end{array}$ & $\frac{\stackrel{n}{g}}{\dot{q}}$ & ڤి & $\begin{array}{l}\infty \\
\stackrel{8}{0} \\
\stackrel{0}{0}\end{array}$ & $\underset{\Xi}{\stackrel{\Xi}{\Xi}}$ \\
\hline & \multirow{3}{*}{ 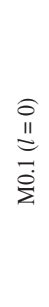 } & $\approx$ & ठ̊ & 蒙 & 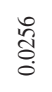 & 守 & 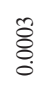 & $\begin{array}{l}\bar{y} \\
\text { ț } \\
0\end{array}$ & 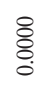 & ᄋ̊̊. & \multirow{4}{*}{ 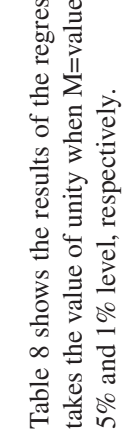 } \\
\hline & & 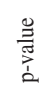 & $\begin{array}{l}5 \\
5 \\
5 \\
0\end{array}$ & $\begin{array}{l}\text { హ్ } \\
\text { రి }\end{array}$ & $\underset{0}{\stackrel{O}{\Xi}}$ & $\begin{array}{l}\text { त్ } \\
\text { రิ }\end{array}$ & $\underset{\substack{\infty \\
0}}{\overrightarrow{0}}$ & 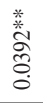 & 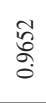 & $\begin{array}{l}20 \\
0 \\
\infty \\
0 \\
0\end{array}$ & \\
\hline & & $\infty$ & 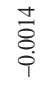 & $\begin{array}{l}\vec{n} \\
\text { 号 } \\
0\end{array}$ & $\begin{array}{l}\mathscr{0} \\
\stackrel{0}{\circ} \\
\dot{0}\end{array}$ & $\begin{array}{l}\bar{\infty} \\
\stackrel{5}{0} \\
0\end{array}$ & $\begin{array}{l}\infty \\
\stackrel{8}{\circ} \\
\stackrel{\leftrightarrow}{1}\end{array}$ & 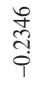 & 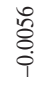 & $\begin{array}{l}\stackrel{\Xi}{a} \\
\stackrel{0}{\oplus}\end{array}$ & \\
\hline & \multicolumn{2}{|c|}{ 总 } & 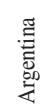 & 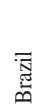 & 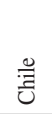 & हू. & 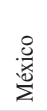 & 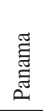 & 壳 & 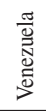 & \\
\hline
\end{tabular}


significance of $10 \%$ and 5\%, respectively. Table 7 contains the results considering the 90-10 interval. The evidence confirms the previous results for the markets of Panama, Mexico and Colombia, all at a statistical significance of $1 \%$. In the case of Peru, the no barrier hypothesis continues to be rejected but now at the 100-level barrier. Finally, Table 8 displays the results in the interval 75-25. The results for Colombia, Mexico and Panama continue to be statistically significant at the conventional levels. In addition, the no barrier hypothesis in rejected by the Argentinian market at the 1000 -level barrier at a statistical significance of $5 \%$. All the other series are either not significant or $\beta$ is not negative.

Overall, evidence suggests that positional effects related to psychological barriers are a relevant phenomenon for the markets of Panama at the lowest barrier level and for the markets of Colombia, Mexico and Peru at the 1000-level barrier. There is weak evidence of barriers in the markets of Argentina, Brazil and Venezuela and practically no evidence at all of psychological barriers around round numbers in the Chilean stock market.

\subsubsection{Barrier Hump Test}

Table 9 shows the results for the barrier hump test, which is meant to test the entire shape of the distribution of M-values. Assuming it should follow a hump-shape distribution, we thus expected $\gamma$ to be negative and significant in the presence of barriers. The results of the barrier hump test partially confirm the evidence presented previously with the barrier proximity tests. The relevance of the psychological barrier of the markets of Panama and Peru, respectively at the lowest barrier level and at the 100-level barrier, is corroborated by the rejection of the no barrier hypothesis at a statistically significant level of $1 \%$. Furthermore, those two markets also reject that hypothesis at the 10-level barrier but only at a statistically significant level of $10 \%$. In the case of the Mexican market, the evidence suggests again that there was a significant barrier at the 1000-level. In addition, the no barrier hypothesis is rejected by the Argentinian market at the 1000- and at the 10000- level barriers, at a statistical significance level of $1 \%$ and $10 \%$, respectively. Finally, there is an indication of a price barrier at the 100-level barrier for the stock market of Venezuela at a statistical significant level of $10 \%$. All the other series are either not significant or $Y$ is not negative.

Overall, from the results presented so far it is possible to discern substantial differences in the incidence of psychological barriers on the markets under study. In the Chilean stock market, it was not detected practically any evidence of psychological barriers. In the case of the stock markets of Argentina, Brazil, Colombia and Venezuela there is weak to mild evidence of barriers mostly at high levels (1000- and 10000-level barriers). Lastly, the stock markets of Mexico, Panama and Peru stock market exhibit the strongest indications of psychological barriers nearby round numbers. In the case of the Panamanian market the positional effect is particularly noticeable at the lowest 
barrier level. In the cases of Peru and Mexico the barrier is especially salient at the 100-level and at the 1000-level, respectively.

\subsubsection{Conditional Effects Test}

Assuming the existence of psychological barriers, we expected the dynamics of return series to be different around these points. In fact, results in Table 10 provide some interesting evidence of mean effects after both upward and downward movements through potential psychological barriers. The coefficients of BD are negative for all indices except Venezuela, and statistically significant for the markets of Argentina, Chile, Colombia, and Peru in the 5-day window around the potential barrier. When the time-window is widened to cover a 10-day period, the effect becomes statistically significant for all but two stock markets (Brazil and Venezuela). This suggests that stock market returns tend to be significantly lower when a barrier is to be crossed on a downward movement. BU is positive and significant for three markets of the sample (Argentina, Brazil, and Peru) considering a 5-day window and for four markets in a 10-day time window (Mexico is added to the three previously mentioned markets). This means that stock markets returns in these countries showed higher returns in the proximity of a barrier when the barrier was to be crossed in an upward movement. The return effects after the breaching of a barrier either on a downward or in an upward movement do not seem to be so clear across the markets. The pattern of conditional effects before the crossing of a possible barrier is similar to that obtained by Cyree et al. (1999) for the Dow Jones, the S\&P500, and other six stock indices.

Table 11 contains results for the conditional variance equations. The variance effects are shown to be particularly evident in a 5-day window before and after an upward movement through a barrier. The coefficient of BU in the variance equation is negative and statistically significant in five of the markets under study. The Peruvian market is the exception with a positive and significant value in BU. This result indicates that in general the markets tend to calm before having risen through a barrier. This is in sharp contradiction with the results obtained by Cyree et al. (1999) according to which, in most cases, markets tend to be more volatile before crossing a barrier in an upward movement. In the post-crossing period, the results are somewhat heterogeneous: three markets present positive and significant results but four other markets show negative and significant coefficients at the conventional levels. Considering a 10-day window, the results are qualitatively similar. The volatility effects around a possible barrier when prices move on a downward movement are not so clear across the markets. Before crossing the barrier prices seem to be less volatile solely in the cases of Argentina and Panama in the 5-day window and in the markets of Colombia, Panama and Venezuela in the 10-day window. After crossing the barrier prices show less volatility in the market of Panama but more volatility in the Colombian market considering a 5-day window. With a longer time window, prices tend to be less volatile not only in Panama but also in Mexico and Venezuela. 


\begin{tabular}{|c|c|c|c|c|c|c|c|c|c|c|}
\hline \multirow{16}{*}{ 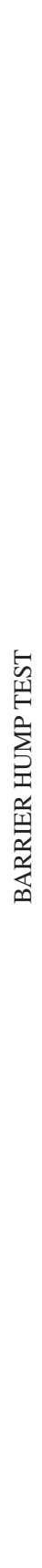 } & \multirow{3}{*}{$\begin{array}{l}\stackrel{+}{+1} \\
\stackrel{\text { II }}{8} \\
\stackrel{8}{\Sigma}\end{array}$} & $\approx$ & $\begin{array}{l}\infty \\
\stackrel{\infty}{0} \\
0\end{array}$ & 훙 & 1 & 1 & $\begin{array}{l}\bar{\infty} \\
0 \\
0 \\
0\end{array}$ & 1 & 1 & $\begin{array}{l}\text { مे } \\
\text { के }\end{array}$ \\
\hline & & 之 & $\stackrel{\text { Oे }}{\circ}$ & 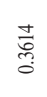 & 1 & 1 & 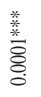 & , & 1 & $\begin{array}{l}\text { శ్ } \\
\text { مิ }\end{array}$ \\
\hline & & $=$ & 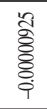 & 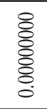 & 1 & 1 & 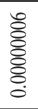 & 1 & 1 & 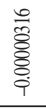 \\
\hline & \multirow{3}{*}{$\begin{array}{l}\text { त) } \\
\stackrel{\text { II }}{\Xi} \\
\stackrel{0}{\Sigma}\end{array}$} & $\approx$ & $\begin{array}{l}\text { 号 } \\
\text { s. }\end{array}$ & $\overline{\bar{\sigma}}$ & $\begin{array}{l}\text { है } \\
\text { t. } \\
0\end{array}$ & 誉 & 号 & 1 & $\begin{array}{l}\not{o} \\
\stackrel{0}{0} \\
0\end{array}$ & ळั \\
\hline & & L & 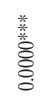 & 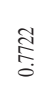 & 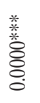 & $\frac{y}{n}$ & 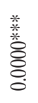 & , & 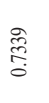 & క్̊ \\
\hline & & $=$ & 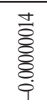 & $\begin{array}{l}\bar{s} \\
\overline{0} \\
\text { o. } \\
0\end{array}$ & 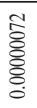 & 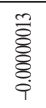 & 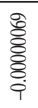 & 1 & 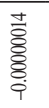 & 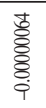 \\
\hline & \multirow{3}{*}{$\begin{array}{l}\text { I } \\
\stackrel{\text { In }}{0} \\
\stackrel{\Xi}{\Sigma}\end{array}$} & $\approx$ & $\begin{array}{l}\stackrel{\infty}{\Xi} \\
0\end{array}$ & 营 & & 导 & 密 & 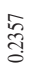 & $\frac{\text { 导 }}{0}$ & ڤ్ \\
\hline & & 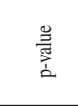 & $\begin{array}{l}\text { 姜 } \\
\text { 号 } \\
\text { 。 }\end{array}$ & $\stackrel{n}{\bar{n}}$ & $\frac{\bar{\Xi}}{0}$ & $\begin{array}{l}\text { ö } \\
\stackrel{0}{0} \\
0\end{array}$ & 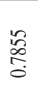 & $\frac{8}{0}$ & 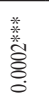 & $\frac{\$}{\circ}$ \\
\hline & & $=$ & 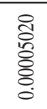 & 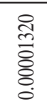 & 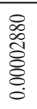 & 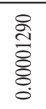 & 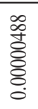 & 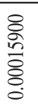 & 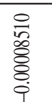 & 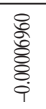 \\
\hline & \multirow{3}{*}{$\begin{array}{l}\widehat{\bar{I}} \\
\stackrel{\Sigma}{\Xi}\end{array}$} & $\approx$ & 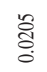 & శ్రి & 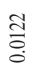 & $\frac{\infty}{0}$ & 僉 & 莕 & $\begin{array}{l}\approx \\
\text { ठ̊. } \\
0\end{array}$ & 旁 \\
\hline & & 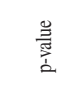 & $\frac{0}{\frac{n}{0}}$ & $\frac{\infty}{a}$ & $\begin{array}{l}\text { Iี } \\
\text { fo }\end{array}$ & $\begin{array}{l}\text { 总 } \\
\stackrel{0}{0}\end{array}$ & ત્તે & 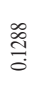 & 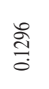 & 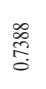 \\
\hline & & $=$ & 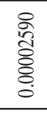 & 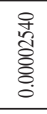 & 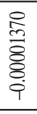 & 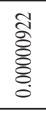 & 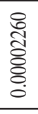 & 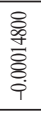 & 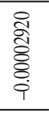 & 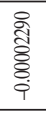 \\
\hline & \multirow{3}{*}{$\begin{array}{l}\stackrel{a}{0} \\
\stackrel{\text { II }}{=} \\
\stackrel{0}{\Sigma}\end{array}$} & $\approx$ & 芯 & $\frac{8}{0}$ & 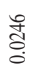 & $\frac{\tilde{n}}{0}$ & 管 & $\begin{array}{l}\tilde{E} \\
\text { 菅 }\end{array}$ & $\begin{array}{l}+ \\
\\
0\end{array}$ & ఫ్ \\
\hline & & $\begin{array}{l}\text { 韋 } \\
\text { 竞 }\end{array}$ & 总 & $\begin{array}{l}\text { : } \\
\text { ర్: } \\
0\end{array}$ & 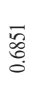 & 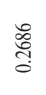 & క్రం & $\begin{array}{l}\frac{\text { 丵 }}{0} \\
\stackrel{0}{0}\end{array}$ & $\begin{array}{l}\stackrel{\mathscr{ٌ}}{\circ} \\
\stackrel{5}{0}\end{array}$ & 恶 \\
\hline & & $=$ & 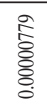 & 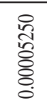 & 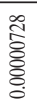 & 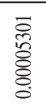 & 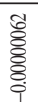 & 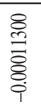 & 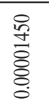 & 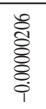 \\
\hline & & .ֶ. & 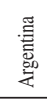 & $\begin{array}{c}\overline{\mathbb{g}} \\
\overline{\mathscr{m}}\end{array}$ & 号 & $\begin{array}{l}\frac{.0}{0} \\
\frac{\overline{2}}{0} \\
\frac{0}{0}\end{array}$ & 这 & 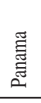 & ᄅ̃ & 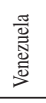 \\
\hline
\end{tabular}




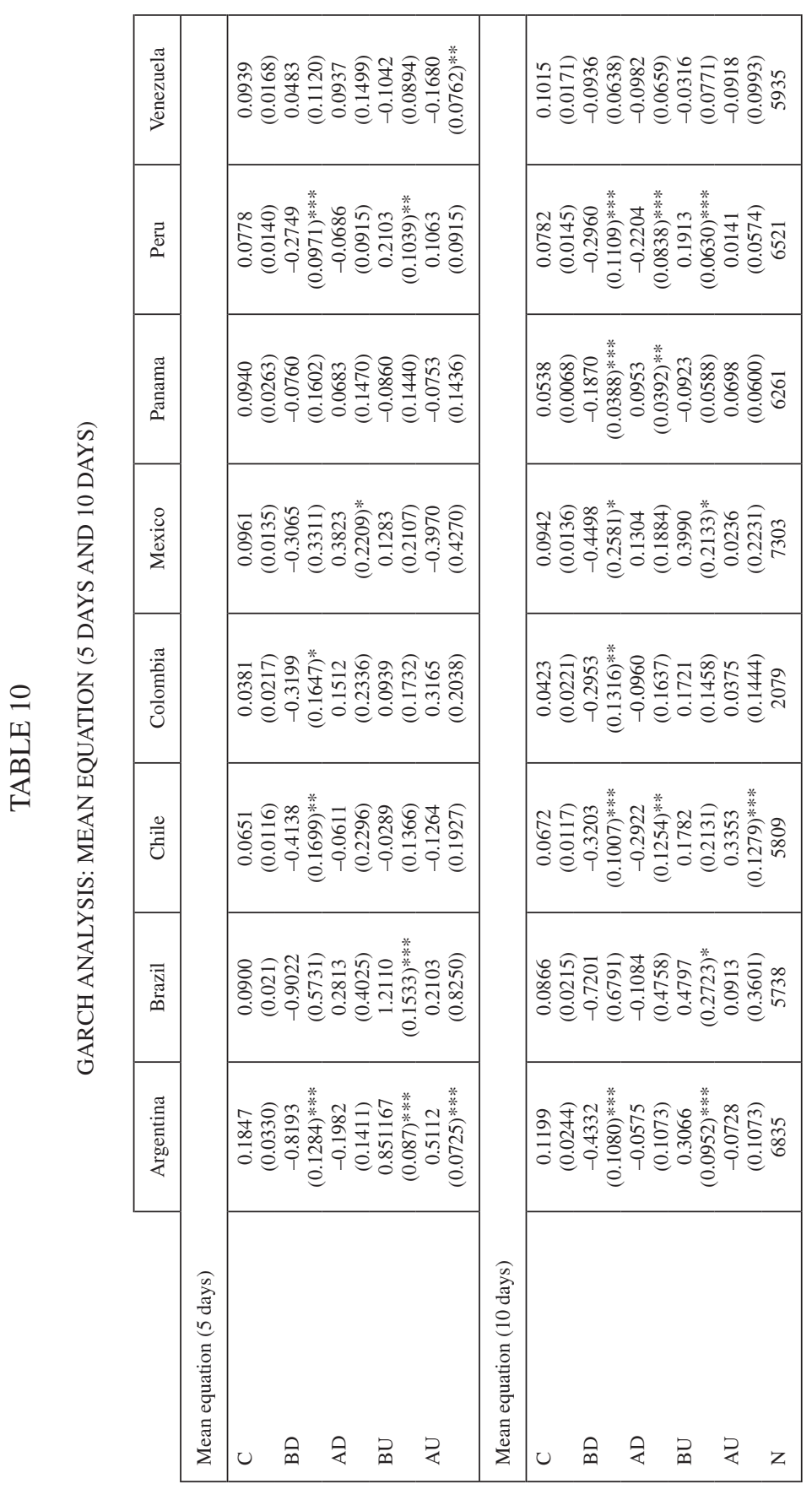

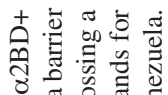

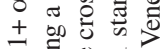
ชे ज्ञ

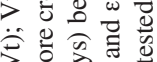

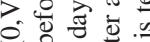
$\sum_{i}$

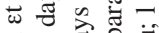

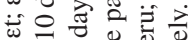
+ in

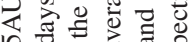
है in tᄒ $\pi$ के + 我

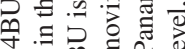

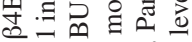

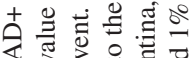

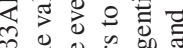
产焉离离。

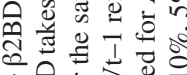
土命志声

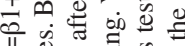
픈 胥焉 。 운음 Ð

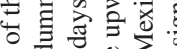
.

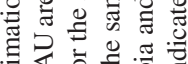

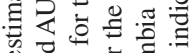
के $\vec{\Xi}: \underline{0}$

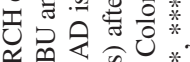

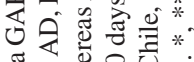

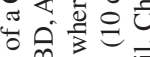

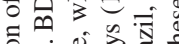

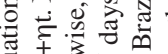
코월

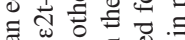
ॠิ $\omega$. 额: I $N \cong$.

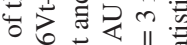

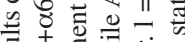

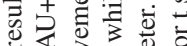

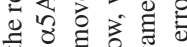

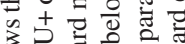
o n。 0 过

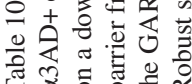




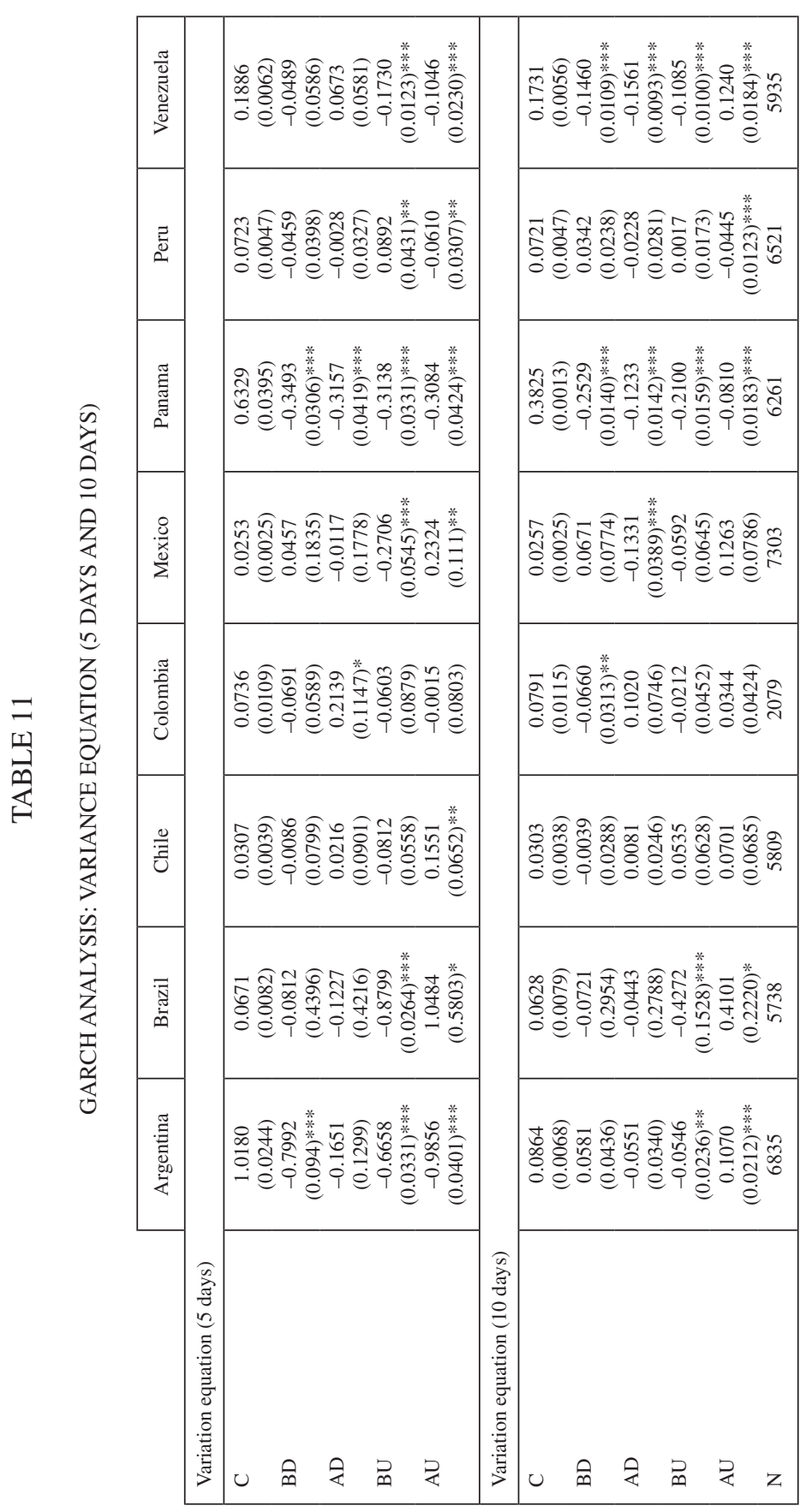

南.

$+\pi$ 元

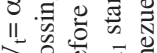

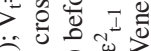

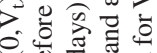

$\sum_{1} 00$

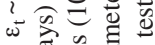

is

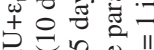

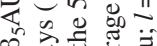

ณ

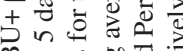

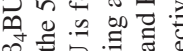

○

吉田墕

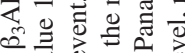

+7 势 0

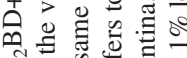

की

क光士腿元

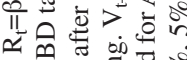

웜

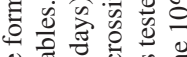

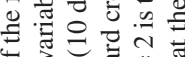

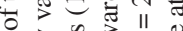

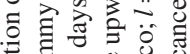

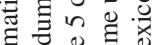

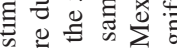

के जे है

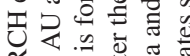

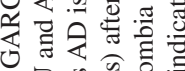

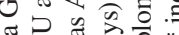

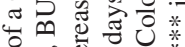

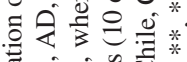

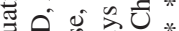

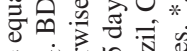

ठำ

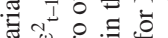

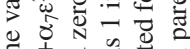
+ +2.3

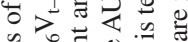

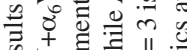

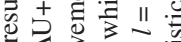

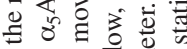

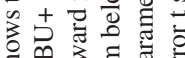

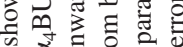

$=0$ 言㝴焉

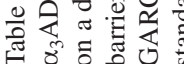


A market by market analysis reveals that the conditional effects were felt, albeit with different intensities, in most of the markets that make up our sample. Regarding the mean effects, two markets deserve to be highlighted due to the intensity and statistical significance of the respective results: the Argentinian market where, within a 5-day period, returns increased in the immediate vicinity of a potential barrier when the latter was crossed in an upward direction, and the Peruvian market, where, within a 10-day period, it was observed a decrease in returns around a potential barrier when it was crossed on a downward movement.

Regarding the effects on volatility, three markets deserve to be mentioned as they had shown a statistically significant reduction of volatility around potential barriers, at a statistically significant level of $1 \%$ : we are referring to the stock markets of Argentina, Panama and Venezuela. In the case of the Panamanian market, the effects were more extensive as they took place before and after the crossing of a barrier, regardless of the direction in which it was to be crossed. For the remaining two markets, the decrease in volatility was especially felt in the vicinity of the barrier when it was to be traversed on an upward movement. In the case of the Argentinian market it is worth noting that the decrease in volatility in these circumstances was accompanied, as already mentioned, by a contemporaneous increase in mean returns. A similar result was obtained by Cyree et al. (1999) for several indices. These authors noticed that this result appeared to represent an "aberration" in the equilibrium risk-return relationship and posed a challenge to the standard financial models that predict a positive correlation between these two variables.

Tables 12 and 13 contain the test results of the four barrier hypothesis mentioned in section 3.2.5, considering a 5-day period and a 10-day period, respectively. If some kind of barrier indeed existed, we would expect that the restraints in terms of mean and variance would be relaxed after the price crossed that barrier.

The results show that the conditional differences in the mean returns and variances tend to be more significant within a 5-day period. In fact, while in the 5-day period there are 10 observations with significant differences at a statistically significant level of $1 \%$ whereas in the 10 -day period there are only three observations with significant differences at the same level of statistical significance.

However, these effects are not uniform across the series under test. In the shorter time horizon, the cases of Argentina and Panama are worth noting since in these two markets there were significant differences in the mean return and in the variance after crossing a barrier both as part of an upward move and as part of a downward move. The Brazilian stock market also shows a significant increase in variance after breaching a barrier no matter the direction of the movement (the results are significant at least at a statistically significant level of 5\%). In general, all these findings are in line with our previous analysis (see Tables 10 and 11).

When we consider a longer time window, of 10 days, the results are weaker and highly significant conditional differences before and after the crossing of a barrier seem 


\begin{tabular}{|c|c|c|c|c|c|}
\hline \multirow{9}{*}{ 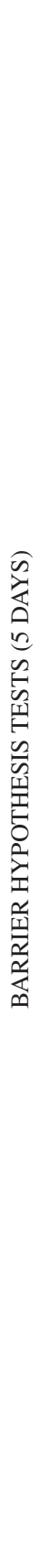 } & $\begin{array}{l}\frac{\pi}{0} \\
\stackrel{0}{N} \\
\stackrel{0}{0}\end{array}$ & \begin{tabular}{ll} 
& \multirow{2}{0}{} \\
8 & 0 \\
0 & $\infty$ \\
0 & 0
\end{tabular} & 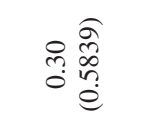 & ñ & 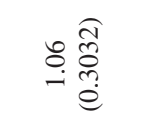 \\
\hline & 哥 & $\stackrel{\overbrace{}}{i} \frac{\overparen{n}}{\stackrel{m}{e}}$ & 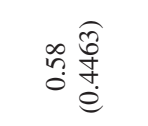 & 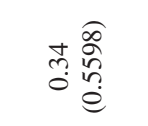 & 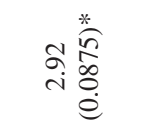 \\
\hline & 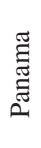 & 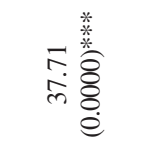 & 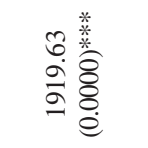 & 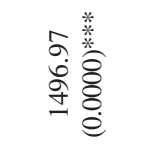 & 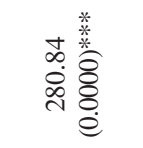 \\
\hline & 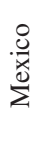 & 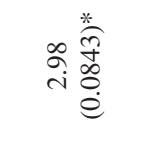 & $\stackrel{\frac{\sigma}{6}}{\frac{\sigma}{e}}$ & 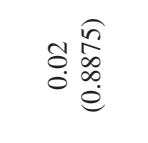 & 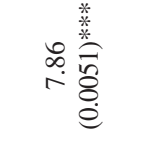 \\
\hline & $\begin{array}{l}\frac{\pi}{0} \\
\frac{0}{0} \\
\frac{0}{0}\end{array}$ & 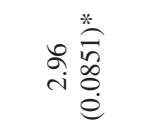 & 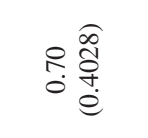 & 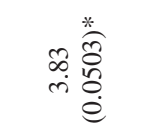 & 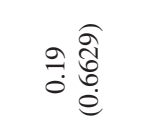 \\
\hline & 节 & 등 $\frac{\stackrel{f}{+}}{\stackrel{+}{巳}}$ & $\begin{array}{l}\underset{\hat{\approx}}{ \pm} \\
\stackrel{0}{0} \\
\dot{0}\end{array}$ & 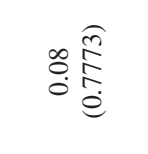 & 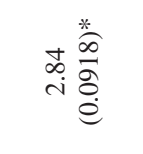 \\
\hline & : & 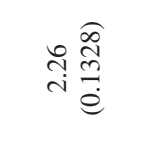 & 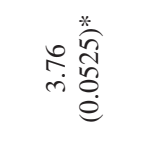 & 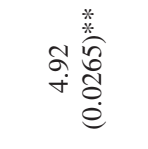 & 气 \\
\hline & 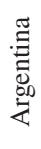 & 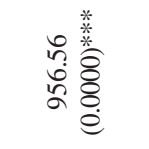 & 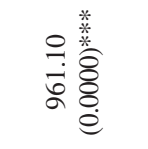 & 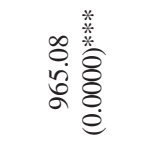 & 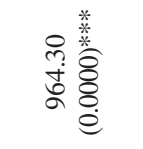 \\
\hline & & 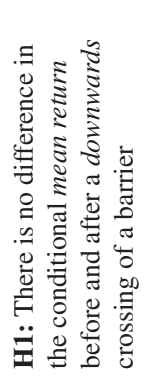 & 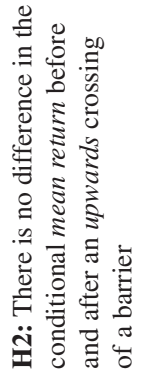 & 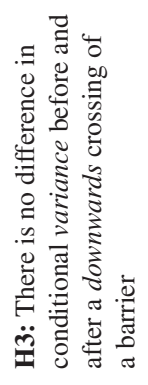 & 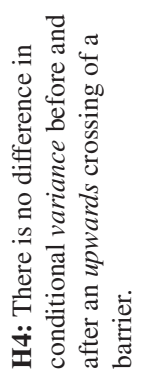 \\
\hline
\end{tabular}




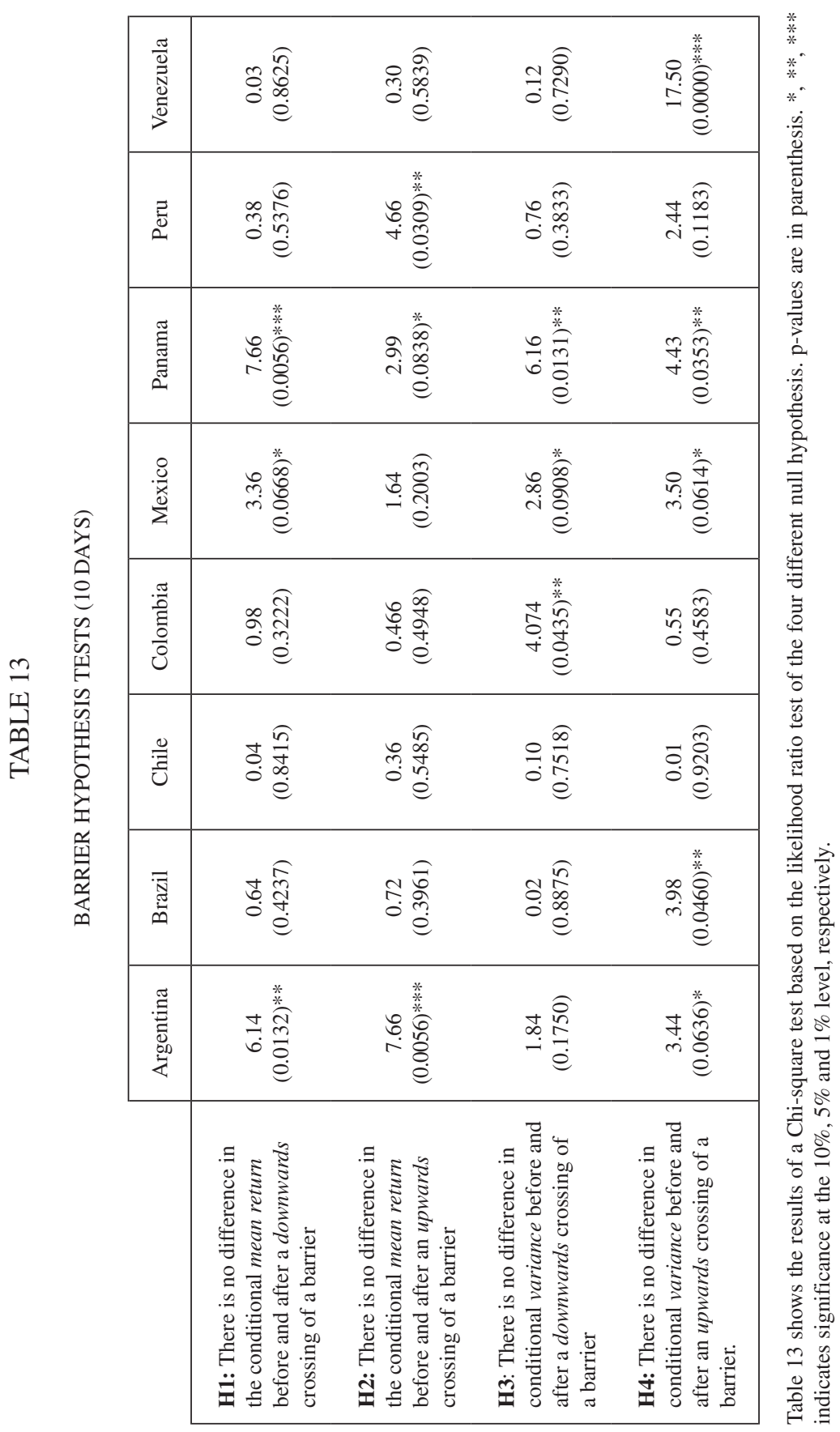


to be observed mainly in the markets of Argentina (in the case of the mean returns) and Panama (both for the mean returns and for the variance). In both cases, there was a significant increase in the mean return and in the variance in the post-crossing period. Interestingly, the market of Chile is the only one that does not exhibit any significant differences in mean returns and variance effects between pre-crossing and post-crossing periods.

Overall, the results obtained reveal substantial differences in the incidence of psychological barriers on the markets of the sample. In fact, when we consider the combination of positional and transgressional effects it is possible to distinguish several different situations. Of all the stock markets under analysis, there is only one -the Chilean market- that has revealed virtually no signs of psychological barriers. Second, there are markets that have shown moderate signs of psychological barriers. We may include in this group the markets of Brazil, Colombia, and Venezuela. In all these cases, the results show that there were some relevant positional effects (case of Colombia) or some relevant transgressional effects (case of Brazil) or a combination between moderate effects of these two types of results (case of Venezuela). Third, the markets of Argentina, Mexico, and Peru can be included in a group where the evidence of psychological barriers is strong. In this instance, the detected positional effects were strong (as in the case of Mexico and Peru) or there was a combination of moderate positional effects with strong transgressional effects (case of Argentina). Finally, it can be concluded that the Panama stock market was the one that showed the strongest signs of psychological barriers in all applied tests (uniformity tests, barrier tests and conditional effects tests).

\section{CONCLUSION}

Psychological barriers have been found to impact financial markets in different geographies and asset classes. Due to several behavioral biases and the consequent inability to make fully rational decisions, the average market practitioner is often affected, directly or indirectly, by such phenomenon.

Following the most widely used methodologies for studying psychological barriers, we provide new evidence regarding this phenomenon in eight Latin American stock markets. Considering an extended sample period, we examined the existence of barriers at round numbers in the major stock market indices of Argentina (MERVAL), Brazil (BOVESPA), Chile (IPSA), Colombia (CALCAP), Mexico (IPC), Panama (BVPSI), Peru (IGBVL), and Venezuela (IBVC).

In summary, it was possible to distinguish three types of situations regarding the presence of positional effects and transgressional effects in the stock markets under scrutiny. First, the Chilean stock market showed virtually no signs of psychological barriers. Second, there is mild evidence of barriers in the cases of the markets 
of Brazil, Colombia and Venezuela. Lastly, the markets of Argentina, Mexico, Panama and Peru showed strong or very strong signs of psychological barriers. The Panamanian market is the one with the strongest indications of psychological barriers nearby round numbers.

The markets of Argentina. Colombia and Mexico seem to present important psychological barriers at the 1000-level barrier while the market of Panama tends to reject the no barrier hypothesis at the lowest level (1-level barrier).

It is interesting to notice that the country with the highest GDP per capita from the countries of the sample and where the stock market presents the largest market capitalization in percentage of the GDP (World Bank, 2016), i.e. that of Chile, is the only one that essentially exhibits no signs of psychological barriers.

The literature on psychological barriers suggests that cultural factors may contribute to this phenomenon (e.g., Cai, Cai and Keasey, 2007). However, in the case of our results, the existence of very different levels of incidence of psychological barriers in countries with relatively similar cultural characteristics suggests that other factors may be relevant. It would be interesting to investigate, in addition to the microstructure characteristics of the markets, the role played by recent economic history in the formation of psychological barriers. Some of the countries in our sample (Argentina, Brazil, and Venezuela, for example) experienced recent historical periods marked by very high levels of inflation. In theory, investors would be expected to become less sensitive to price barriers in countries where the nominal level of prices increases very rapidly. However, the results presented in this article do not confirm this conjecture.

Overall, these findings provide evidence supporting the existence of psychological barriers with respect to index returns. Our results are thus in line with earlier studies (e.g., Koedijk and Stork, 1994; Cyree et al., 1999; Bahng, 2003; Woodhouse et al., 2016) and can be regarded as evidence that prices tend to exhibit some pockets of predictability thus contradicting the random walk usually associated with efficient capital markets. Moreover, our results seem to suggest that technical analysis strategies based on price support and resistances around round numbers can be profitable, at least in some stock markets. However, it should be noted that the fact that prices may be predictable, in itself, does not necessarily mean that investors should be able to obtain abnormal profits. The existence of significant limits to arbitrage (arising from synchronization risk, noise trader risk or trading costs, for example) may prevent investors from profiting from predictable stock prices.

There is much to be investigated about psychological barriers in financial markets. Further avenues for research may include the analysis of trading volume around possible price barriers, the adoption of statistical tests based on the assumption that prices follow specific distributions (e.g., the Benford' Law) and the study of the impact of salient events (e.g., a financial crisis) on the prevalence of price barriers. 


\section{REFERENCES}

AGGARWAL, R.; LUCEY, B.M. (2007). Psychological barriers in gold prices? Review of Financial Economics, 16, 217-230.

BAHNG, S. (2003). Do Psychological Barriers Exist in the Stock Price Indices? Evidence from Asia's Emerging Markets. International Area Studies Review, 6, 35-52.

BERK, A.S.; CUMMINS, M.; DOWLING, M.; LUCEY, B.M. (2017). Psychological Price Barriers in Frontier Equities. Journal of International Financial Markets, Institutions \& Money, 49, 1-14.

BERTOLA G.; CABALLERO, R.J. (1992). Target Zones and Realignments. American Economic Review, $82,520-536$.

BROCK, W.; LAKONISHOK, J.; LEBARON, B. (1992). Simple technical trading rules and the stochastic properties of stock returns. Journal of Finance, 47, 1731-1764.

BURKE, S. (2001). Barriers in U.S. Benchmark Bonds. Unpublished manuscript. Vancouver.

CAI, B. M.; CAI, C.X.; KEASEY, K. (2007). Influence of cultural factors on price clustering and price resistance in China's stock markets. Accounting and Finance, 47, 623-64.

CARRERA, C. (2015). Tracking exchange rate management in Latin America. Review of Financial Economics, 25, 35-41.

CHEN, M.; TAI, V.W. (2011). Psychological Barriers and Prices Behaviour of TAIFEX Futures. Global Economy and Finance Journal, 4, 1-12.

CYREE, K.B.; DOMIAN, D.L.; LOUTON, D.A.; YOBACCIO, E.J. (1999). Evidence of psychological barriers in the conditional moments of major world stock indices. Review of Financial Economics, 8, 73-91.

De CEUSTER, M.J.K.; DHAENE, G.; SCHATTEMAN, T. (1998). On the hypothesis of psychological barriers in stock markets and Benford's Law. Journal of Empirical Finance, 5, 263-279.

De GRAUWE, P.; DECUPERE, D. (1992). Psychological barriers in the foreign exchange markets. Journal of International and Comparative Economics, 1, 87-101.

DONALDSON, R.G. (1990a). Psychological barriers in asset prices, rationality and the efficient market hypothesis. Princeton Financial Research Center Memorandum, 114.

DONALDSON, R.G. (1990b). International evidence on psychological barriers in asset prices and the efficient market hypothesis. Princeton Financial Research Center Memorandum, 116.

DONALDSON, R.G.; KIM, H.Y. (1993). Price Barriers in the Dow Jones Industrial Average. Journal of Financial and Quantitative Analysis, 28, 313-330.

DORFLEITNER, G.; KLEIN, C. (2009). Psychological barriers in European stock markets: Where are they? Global Finance Journal, 19, 268-285.

DOWLING, M.; CUMMINS, M.; LUCEY, B.M. (2016). Psychological barriers in oil futures markets. Energy Economics, 53, 293-304.

FONSECA, V.; PACHECO, L.; LOBÃO, J. (2020). Psychological barriers in the cryptocurrency market. Review of Behavioral Finance, forthcoming, DOI: 10.1108/RBF-03-2019-0041

GEORGE, T.J.; HWANG, C. (2004). The 52-Week High and Momentum Investing. Journal of Finance. $59,2145-2176$.

JANG, B.; KIM, C.; KIM, K.T.; LEE, S.; SHIN, D. (2015). Psychological Barriers and Option Pricing. Journal of Futures Markets, 35, 52-74.

KOEDIJK, K.G.; STORK, P.A. (1994). Should we care? Psychological barriers in stock markets. Economics Letters, 44, 427-432.

LEY, E.; VARIAN, H.R. (1994). Are there psychological barriers in the Dow-Jones index? Applied Financial Economics, 4, 217-224.

LI, X.; LI, X.; CHONG, X. (2020). Price clustering in Bitcoin market - An extension. Finance Research Letters, forthcoming. DOI: 10.1016/j.frl.2018.12.020

LUCEY, M. E.; O'CONNOR, F.A. (2016). Mind the gap: Psychological barriers in gold and silver prices. Finance Research Letters, 17, 135-140.

MITCHELL, J.Ç IZAN, H.Y. (2006). Clustering and psychological barriers in exchange rates. Journal of International Financial Markets, Institutions and Money, 16, 318-344. 
PALAO, F.; PARDO, A. (2018). Do Price Barriers Exist in the European Carbon Market? Journal of Behavioral Finance, 19, 111-124.

SCHWARTZ, A.L.; VAN NESS, B.F.; VAN NESS, R.A. (2004). Clustering in the futures market: Evidence from S\&P 500 futures contracts. Journal of Futures Markets, 24, 413-428.

SHAW, L.K.J.; KALAICHELVAN, M. (2012). A Critical Evaluation of the Significance of Round Numbers in European Equity Markets in Light of the Predictions from Benford's Law. International Research Journal of Finance and Economics, 95, 196-210.

SHILLER, R.J. (2015). Irrational Exuberance. 3rd edition. Princeton University Press, Princeton, NJ.

TVERSKY, A.; KAHNEMAN, D. (1974). Judgment under uncertainty: Heuristics and biases. Science, New Series, 185, 1124-1131.

WESTERHOFF, F. (2003). Anchoring and psychological barriers in foreign exchange markets. Journal of Behavioral Finance, 4, 65-70.

WOODHOUSE, S.A.; SINGH, H.; BHATTACHARYA, S.; KUMAR, K. (2016). Invisible walls: Do psychological barriers really exist in stock index levels? North American Journal of Economics and Finance, 36, 267-278.

WORLD BANK (2016). World Development Indicators Database. World Bank. Washington, DC. 